\title{
Stability analysis of a nonlinear coupled implicit switched singular fractional differential system with $p$-Laplacian
}

\author{
Manzoor Ahmad ${ }^{1}$, Akbar Zada and Jehad Alzabut ${ }^{2 *}$ (I)
}

"Correspondence:

jalzabut@psu.edu.sa

${ }^{2}$ Department of Mathematics and

General Sciences, Prince Sultan

University, Riyadh, Saudi Arabia

Full list of author information is

available at the end of the article

\section{Springer}

\begin{abstract}
This paper deals with existence, uniqueness, and Hyers-Ulam stability of solutions to a nonlinear coupled implicit switched singular fractional differential system involving Laplace operator $\phi_{p}$. The proposed problem consists of two kinds of fractional derivatives, that is, Riemann-Liouville fractional derivative of order $\beta$ and Caputo fractional derivative of order $\sigma$, where $m-1<\beta, \sigma<m, m \in\{2,3, \ldots\}$. Prior to proceeding to the main results, the system is converted into an equivalent integral form by the help of Green's function. Using Schauder's fixed point theorem and Banach's contraction principle, the existence and uniqueness of solutions are proved. The main results are demonstrated by an example.
\end{abstract}

MSC: 26A33; 34A08; 34B27

Keywords: Singular fractional differential equation; Riemann-Liouville fractional derivative; Caputo fractional derivative; Schauder's fixed point theorem; Banach contraction principle; Hyers-Ulam stability

\section{Introduction}

Fractional differential equations (FDEs) arise in different branches of applied mathematics. Recently, it has been evidently realized that the mathematical models of systems and processes involving fractional order derivatives often appear in the fields of physics, chemistry, biology, viscoelasticity, control hypothesis, speculation, fluid dynamics, hydrodynamics, aerodynamics, information processing system networking, notable and picture processing; see the remarkable monographs [22, 24, 37]. The study of fractional order differential models has been associated with the fact that they provide a more accurate description of real phenomena than the counterpart integer order models. The reason behind this intensive interest is that FDEs provide practical tools for the depictions of memory and inherited properties of many materials and processes. As a result, FDEs have experienced significant developments in recent years; see [5, 6, 13, 28, 30, 32, 34, 35, 41, 45] for further details.

One of the most interesting research areas in the field of FDEs, which has attracted great consideration amongst researchers, is dedicated to the existence theory of the solutions of fractional models. The aforesaid part has been extensively explored for integer order differential equations (DEs). However, for arbitrary order DEs, there are still many

(c) The Author(s) 2019. This article is distributed under the terms of the Creative Commons Attribution 4.0 International License (http://creativecommons.org/licenses/by/4.0/), which permits unrestricted use, distribution, and reproduction in any medium, provided you give appropriate credit to the original author(s) and the source, provide a link to the Creative Commons license, and indicate if changes were made. 
aspects which need further study and research. Different mathematicians have explored the existence of solutions of FDEs in various directions [1-3, 9, 12, 17, 21, 27]. Another imperative and more remarkable area of research, which has recently attracted more attention, is committed to the stability analysis of DEs of integer and noninteger order. The first effort was initiated by Ulam himself and later was confirmed by Hyers in [19]. That is why this type of stability is referred to as Ulam-Hyers (UH) stability. Further, Rassias introduced the Ulam-Hyers-Rassias (UHR) stability; see some recently reported stability results in the sense of Ulam $[4,7,20,29,33,38,42-44,47-50]$. It is to be noted that the above said areas of interest (existence and stability) have been often deliberated within the settings of Riemann-Liouville and Caputo derivatives. The above results can also be studied for Caputo-Fabrizio derivative [10, 11, 14-16, 23].

In the solutions of differential and integral equations, the concept of fixed point theory is very important. Different fixed point theorems, which have numerous applications in the mentioned equations, are presented. Few important fixed point theorems can be found in $[18,39,46]$.

For the sake of completeness and comparison, we assemble herein some relevant results. In [26], Liu et al. investigated the existence results of fractional Sturm-Liouville boundary value problem:

$$
\left\{\begin{array}{l}
D_{0^{+}}^{\sigma}(\Phi(\rho(t)))\left(D_{0^{+}}^{\sigma^{\prime}} u(t)\right)+f\left(t, u(t), D_{0^{+}}^{\sigma^{\prime}} u(t)\right)=0, \quad t \in(0,1), 0<\sigma, \sigma^{\prime}<1 \\
a_{0} \lim _{t \rightarrow 0} t^{1-\sigma^{\prime}} u(t)-b_{0} \lim _{t \rightarrow 0} \Phi^{-1}\left(t^{1-\sigma}\right) \rho(t) D_{0^{+}}^{\sigma^{\prime}} u(0)=0 \\
c_{0} \lim _{t \rightarrow 1} \Phi^{-1}\left(t^{1-\sigma}\right) \rho(t) D_{0^{+}}^{\sigma^{\prime}} u(0)+d_{0} \lim _{t \rightarrow 1} t^{1-\sigma^{\prime}} u(t)=0
\end{array}\right.
$$

where $D_{0^{+}}^{\sigma}, D_{0^{+}}^{\sigma^{\prime}}$ denote the Riemann-Liouville fractional derivatives of order $\sigma$ and $\sigma^{\prime}$ respectively, $a_{0}, b_{0}, c_{0}, d_{0} \in \mathcal{R}$, while $\rho:(0,1) \rightarrow \mathcal{R}^{+}$is a given continuous function. The function $f:(0,1) \times \mathcal{R} \times \mathcal{R} \rightarrow \mathcal{R}$ is a quasi-Carathéodory function which may be singular at the points $t=0,1$. The $p$-Laplacian operator $\Phi$ is defined as $\Phi(s)=|s|^{p-2}$ with inverse operator represented by $\Phi^{-1}(s)=|s|^{q-2}$, where $\frac{1}{p}+\frac{1}{q}=1$. The analysis relies on the wellknown Leray-Schauder alternative principle.

In [25], Li studied the existence of a positive solution to the fractional differential equation involving integral boundary conditions with nonlinear $p$-Laplacian operator of the form:

$$
\left\{\begin{array}{l}
D^{\alpha}\left(\phi_{p}\left({ }^{c} D^{\sigma} u(t)\right)\right)+f(t, u(t))=0, \quad t \in(0,1), 2<\alpha, \sigma<3, \\
\phi_{p}\left({ }^{c} D^{\sigma} u(0)\right)=\left[\phi_{p}\left({ }^{c} D^{\sigma} u(0)\right)\right]^{\prime}=\left({ }^{c} D^{\sigma} u(1)\right)=0, \\
u^{\prime \prime}(0)=u^{\prime}(1)=0, \\
\lambda u(0)+\zeta u^{\prime}(0)=\int_{0}^{1} u(t) \varphi(t) d t,
\end{array}\right.
$$

where $D^{\alpha},{ }^{c} D^{\sigma}$ denote the Riemann-Liouville and Caputo fractional derivatives of order $\alpha$ and $\sigma$, respectively, and $\phi(s)=|s|^{p-2}, p>1$. The function $\varphi$ satisfies $\varphi:[0,1] \rightarrow \mathcal{R}^{+}$with $\varphi \in L^{1}[0,1], \int_{0}^{1} \varphi(t) d t>0$ and $\int_{0}^{1} t \varphi(t) d t>0, a, b \in \mathcal{R}^{+}$with $\int_{0}^{1} \varphi(t) d t<a$, where $b>a$, and $f:[0,1] \times(0, \infty) \rightarrow(0, \infty)$ is continuous. By employing the Avery-Henderson fixed point theorem, new results have been obtained for the above problem. 
In [8], Alkhazzan et al. studied the existence and stability results for a class of nonlinear fractional differential equations with singularity of the form:

$$
\left\{\begin{array}{l}
{ }^{c} D^{\sigma}\left[\phi_{p} D^{\beta} u(t)\right]+F_{1}(t) \psi_{1}(t, u(t))=0, \\
\left(\left[\phi_{p} D^{\beta} u(0)\right]\right)^{(j)}=0, \quad j=0,1, \ldots, m-1, \\
I^{k-\beta}(u(0))=0, \quad k=2,3, \ldots, m, \\
D^{\delta}(u(1))=0,
\end{array}\right.
$$

where $D^{\beta}$ and ${ }^{c} D^{\sigma}$ respectively represent the Riemann-Liouville and Caputo fractional derivatives of order $\beta$ and $\sigma, m-1<\beta, \sigma \leq m, m \in\{2,3, \ldots\}, 1<\delta \leq 2$. The nonlinear $p$-Laplacian operator $\phi_{p}$ has expression in the form $\phi_{p}(\theta)=\frac{\theta}{|\theta|^{2-p}}, \phi_{p}(0)=0$, with inverse $\phi_{q}$, that is, $\phi_{q}=\phi_{p}^{-1}$ such that $\frac{1}{p}+\frac{1}{q}=1$. The nonlinear function $\psi_{1} \in \mathcal{C}[0,1]$ is continuous and perhaps singular with respect to $t, u$. Some classical fixed point theorems are utilized to prove the main results.

The objective of this paper is to use the concepts mentioned in [8] to examine the existence, uniqueness as well as different kinds of Hyers-Ulam stability for the solution of the nonlinear coupled implicit switched singular system of fractional differential equations with singularities of the form:

$$
\left\{\begin{array}{l}
\left.{ }^{c} D^{\sigma}\left[\phi_{p} D^{\beta} u(t)\right]+\mathcal{F}_{1}(t) \psi_{1}\left(t, u(t),{ }^{c} D^{\rho}\left[\phi_{p} D^{\beta} v(t)\right]\right)=0, \quad t \in \mathrm{J}=\right] 0,1[ \\
{ }^{c} D^{\rho}\left[\phi_{p} D^{\beta} v(t)\right]+\mathcal{F}_{2}(t) \psi_{2}\left(t,{ }^{c} D^{\sigma}\left[\phi_{p} D^{\beta} u(t)\right], v(t)\right)=0, \quad t \in \mathrm{J}, \\
\left(\left[\phi_{p} D^{\beta} u(0)\right]\right)^{(j)}=0, \quad j=0,1, \ldots, m-1, \\
\left(\left[\phi_{p} D^{\beta} v(0)\right]\right)^{(j)}=0, \quad j=0,1, \ldots, m-1, \\
I^{k-\beta}(u(0))=I^{k-\beta}(v(0))=0, \quad k=2,3, \ldots, m, \\
D^{\delta}(u(1))=D^{\delta}(v(1))=0,
\end{array}\right.
$$

where $D^{\beta}$ and ${ }^{c} D^{\sigma}$ respectively denote the Riemann-Liouville and Caputo fractional derivatives of order $\beta$ and $\sigma, m-1<\beta, \sigma \leq m, m \in\{2,3, \ldots\}, 1<\delta \leq 2$, and $\mathcal{F}_{1}(\cdot), \mathcal{F}_{2}(\cdot)$ are linear and bounded operators on $\mathcal{R}$. Furthermore, the nonlinear $p$-Laplacian operator $\phi_{p}$ has expression in the form $\phi_{p}(\theta)=\frac{\theta}{|\theta|^{2-p}}, \phi_{p}(0)=0$, with inverse operator $\phi_{q}$, that is, $\phi_{q}=\phi_{p}^{-1}$ such that $\frac{1}{p}+\frac{1}{q}=1$. The nonlinear functions $\psi_{1}, \psi_{2} \in \mathcal{C}[0,1]$ are continuous and perhaps singular with respect to $t, u, v$.

The current work is organized as follows: In Sect. 2, we present some basic definitions and assertions that will be used in the subsequent sections. In Sect. 3 we state and prove our main existence results. We discuss the Ulam stability of the proposed problem in Sect. 4. Concrete example is illustrated to demonstrate consistency with the proposed results.

\section{Basic definitions and assertions}

Here we state some fundamental facts, definitions, and lemmas which will be used throughout this paper.

Let $\mathrm{C}(\mathrm{J}, \mathcal{X})$ be the space of all continuous functions of the form $u(t): \mathrm{J} \rightarrow \mathcal{X}, t \in \mathrm{J}$. It is obvious that $\mathrm{C}(\mathrm{J}, \mathcal{X})$ is a Banach space with norm $\|u\|=\max \{|u(t)|, t \in \mathrm{J}\}$. Further, we understand that $\mathrm{C}(\mathrm{J}, \mathcal{X}) \times \mathrm{C}(\mathrm{J}, \mathcal{X})$ is a Banach space with norm $\|(u, v)\|=\|u\|+\|v\|$. 
Definition 2.1 ([22]) Let $\alpha \in \mathcal{R}^{+}$. Then the noninteger order integral in the RiemannLiouville sense for a function $\theta: J \rightarrow \mathcal{R}$ is given as

$$
\mathcal{I}^{\alpha} \theta(t)=\frac{1}{\Gamma(\alpha)} \int_{0}^{t}(t-s)^{\alpha-1} \theta(s) d s
$$

such that the integral on the right-hand side is pointwise defined on $\mathcal{R}^{+}$.

Definition 2.2 ([22]) Let $\alpha \in(n-1, n]$ with $n-1=[\alpha]$. Then the noninteger order derivative in the Caputo sense of $\theta:[a, b] \rightarrow \mathcal{R}$ is stated as

$$
\frac{d^{\alpha}}{d t^{\alpha}} \theta(t)=\int_{a}^{t} \frac{(t-s)^{n-\alpha-1}}{\Gamma(n-\alpha)}\left(\frac{d^{n}}{d s^{n}} \theta(s)\right) d s
$$

In particular, if $p$ is defined on the interval $[a, b]$ and $\alpha \in(0,1]$, then

$$
\frac{d^{\alpha}}{d t^{\alpha}} \theta(t)=\frac{1}{\Gamma(1-\alpha)} \int_{a}^{t} \frac{\theta^{\prime}(s)}{(t-s)^{\alpha}} d s, \quad \text { where } \theta^{\prime}(s)=\frac{d \theta(s)}{d s} .
$$

It is to be noted that the integral on the right-hand side is pointwise defined on $\mathcal{R}^{+}$.

Definition 2.3 ([22]) The noninteger order derivative in the Riemann-Liouville sense having order $\sigma$ for a function $\theta:(0, \infty) \rightarrow \mathcal{R}^{+}$is defined by

$$
D_{1^{+}}^{\sigma} \theta(t)=\frac{1}{\Gamma(n-\sigma)} \frac{d^{n}}{d t^{n}} \int_{0}^{t}(t-s)^{n-\sigma-1} \theta(s) d s, \quad n-1<\sigma<n=1+\lceil\sigma\rceil,
$$

where the integral on the right-hand side is pointwise continuous and defined on the interval $(0, \infty)$ and $\lceil\sigma\rceil$ is the integer part of $\sigma$.

Lemma 2.4 ([8]) Let $\sigma \in(m-1, m], \theta \in \mathcal{C}^{m-1}$, and ${ }^{c} D^{\sigma}$ be the Caputo fractional derivative. Then

$$
{ }^{c} I^{\sigma c} D^{\sigma} \theta(t)=\theta(t)+b_{1}+b_{2} t+b_{3} t^{2}+\cdots+b_{m} t^{m-1},
$$

where $b_{i} \in \mathcal{R}, i=1,2, \ldots m, m=[\sigma]+1$.

Lemma 2.5 ([8]) Let $\sigma \in(m-1, m], \theta \in \mathcal{C}^{m-1}$, and $D^{\sigma}$ be the Riemann-Liouville fractional derivative. Then

$$
I^{\sigma} D^{\sigma} \theta(t)=\theta(t)+c_{1} t^{\sigma-1}+c_{2} t^{\sigma-2}+\cdots+c_{m} t^{\sigma-m}
$$

where $c_{i} \in \mathcal{R}, i=1,2, \ldots m, m=[\sigma]+1$.

Lemma 2.6 ([36], Arzelä-Ascoli theorem) An operator $\mathcal{H}: \mathcal{B}_{r} \cap\left(\bar{\Omega}_{1} / \Omega_{2}\right) \rightarrow \mathcal{B}_{r}$ is said to be compact if and only if $\mathcal{H}$ is uniformly bounded and discontinuous.

Lemma 2.7 (Schauder fixed point theorem [39]) Let $\mathcal{S} \neq \emptyset$ be a convex and closed subset of the Banach space $\mathcal{X}$. Let $\phi: \mathcal{S} \rightarrow \mathcal{S}$ be a continuous operator such that $\phi(\mathcal{S})$ is a relatively compact subset of $\mathcal{X}$. Then the operator system $\phi$ has at least one fixed point in $\mathcal{S}$. 
Lemma 2.8 ([27]) Let $\phi_{p}: \mathcal{R} \rightarrow \mathcal{R}$ be a nonlinear $p$-Laplacian operator, that is, $\phi_{p}(\zeta)=$ $|\zeta|^{p-2} \zeta, \zeta \in \mathcal{R}$. Then

$$
\frac{d \phi_{p}}{d \zeta}=(p-1)|\zeta|^{p-2}
$$

Some basic properties of the operator $\phi_{p}$ are as follows:

$\left(\mathcal{A}_{1}\right)$ If $1<p \leq 2, \zeta_{1}, \zeta_{2}>0,0<\varrho \leq\left|\zeta_{1}\right|,\left|\zeta_{2}\right|$, then

$$
\left|\phi_{p}\left(\zeta_{1}\right)-\phi_{p}\left(\zeta_{2}\right)\right| \leq(p-1) \varrho^{p-2}\left|\zeta_{1}-\zeta_{2}\right|
$$

$\left(\mathcal{A}_{2}\right)$ For $p>2,\left|\zeta_{1}, \zeta_{2}\right| \leq \varrho *$, then

$$
\left|\phi_{p}\left(\zeta_{1}\right)-\phi_{p}\left(\zeta_{2}\right)\right| \leq(p-1) \varrho^{p-2}\left|\zeta_{1}-\zeta_{2}\right|
$$

Lemma 2.9 ([31]) Let $\mathcal{X}$ be a Banach space and $\mathcal{B} \subset \mathcal{X}$ be a nonempty, closed, and convex set. If a map $\mathcal{H}: \mathcal{B} \rightarrow \mathcal{B}$ is compact, then $\mathcal{H}$ has a fixed point.

Definition 2.10 (Urs [40], Definition 2) Let $\mathcal{X}$ be a Banach space such that $\Upsilon_{1}, \Upsilon_{2}: \mathcal{X} \times$ $\mathcal{X} \rightarrow \mathcal{X}$ are two operators. Then the system

$$
\left\{\begin{array}{l}
y(t)=\Upsilon_{1}(y, z)(t), \\
z(t)=\Upsilon_{2}(y, z)(t),
\end{array}\right.
$$

is said to be Hyers-Ulam stable if there exist constants $\digamma_{j}(j=1,2,3,4)>0$ with $\alpha_{j}(j=1,2)>$ 0 , and for each solution $\left(y^{*}, z^{*}\right) \in \mathcal{X} \times \mathcal{X}$ of the inequalities

$$
\left\{\begin{array}{l}
\left\|y^{*}-\Phi\left(y^{*}, z^{*}\right)\right\| \leq \alpha_{1} \\
\left\|z^{*}-\Psi\left(y^{*}, z^{*}\right)\right\| \leq \alpha_{2}
\end{array}\right.
$$

there exists a solution $\widetilde{y}, \widetilde{z}) \in \mathcal{X} \times \mathcal{X}$ of system (2.1), which satisfies

$$
\left\{\begin{array}{l}
\left\|y^{*}-\tilde{y}\right\| \leq \digamma_{1} \alpha_{1}+\digamma_{2} \alpha_{2}, \\
\left\|z^{*}-\tilde{z}\right\| \leq \digamma_{3} \alpha_{1}+\digamma_{4} \alpha_{2} .
\end{array}\right.
$$

Definition 2.11 Let $v_{J}$, where $J=1,2, \ldots, k$, be the eigenvalues (real or complex) of a matrix $\digamma \in \varpi^{k \times k}$. Then the term spectral radius $\alpha(\digamma)$ of $\digamma \in \varpi^{k \times k}$ is defined as

$$
\alpha(\digamma)=\max \left\{\left|v_{J}\right| \text { for } J=1,2, \ldots, k\right\} .
$$

It is well known that the system corresponding to matrix $\digamma \in \varpi^{k \times k}$ will converge to zero if $\alpha(\digamma)$ is less than one.

Theorem 2.12 (Urs [40], Theorem 4) Consider a Banach space $\mathcal{X}$ and define two operators $\Upsilon_{1}, \Upsilon_{2}: \mathcal{X} \times \mathcal{X} \rightarrow \mathcal{X}$ such that

$$
\left\{\begin{array}{l}
\left\|\Upsilon_{1}(\mathbf{y}, \mathbf{z})-\Upsilon_{1}\left(\mathbf{y}^{*}, \mathbf{z}^{*}\right)\right\| \leq \digamma_{1}\left\|\mathbf{y}-\mathbf{y}^{*}\right\|+\digamma_{2}\left\|\mathbf{z}-\mathbf{z}^{*}\right\|, \\
\left\|\Upsilon_{2}(\mathbf{y}, \mathbf{z})-\Upsilon_{2}\left(\mathbf{y}^{*}, \mathbf{z}^{*}\right)\right\| \leq \digamma_{3}\left\|\mathbf{y}-\mathbf{y}^{*}\right\|+\digamma_{4}\left\|\mathbf{z}-\mathbf{z}^{*}\right\| .
\end{array}\right.
$$


If the spectral radius of the matrix

$$
\digamma=\left(\begin{array}{ll}
\digamma_{1} & \digamma_{2} \\
\digamma_{3} & \digamma_{4}
\end{array}\right)
$$

is less than one, then the fixed points correlated with operating system (2.1) are Hyers-Ulam stable.

\section{Existence results}

This section is devoted to investigating the existence of solutions. The first result converts the proposed problem into an equivalent integral form by the help of Green's function.

Theorem 3.1 Let $\psi_{1}, \psi_{2}$ be integrable functions and $u, v \in C(J, \mathcal{X})$ satisfy (1.1). Then, for $3<\sigma, \beta, \rho \leq m, m \geq 4$, the solution of the switched coupled system

$$
\left\{\begin{array}{l}
{ }^{c} D^{\sigma}\left[\phi_{p} D^{\beta} u(t)\right]+\mathcal{F}_{1}(t) \psi_{1}\left(t, u(t),{ }^{c} D^{\rho}\left[\phi_{p} D^{\beta} v(t)\right]\right)=0, \quad t \in \mathrm{J}, \\
{ }^{c} D^{\rho}\left[\phi_{p} D^{\beta} v(t)\right]+\mathcal{F}_{2}(t) \psi_{2}\left(t,{ }^{c} D^{\sigma}\left[\phi_{p} D^{\beta} u(t)\right], v(t)\right)=0, \quad t \in \mathrm{J}, \\
\left(\left[\phi_{p} D^{\beta} u(0)\right]\right)^{(j)}=0, \quad j=0,1, \ldots, m-1, \\
\left(\left[\phi_{p} D^{\beta} v(0)\right]\right)^{(j)}=0, \quad j=0,1, \ldots, m-1, \\
I^{k-\beta}(u(0))=I^{k-\beta}(v(0))=0, \quad k=2,3, \ldots, m, \\
D^{\delta}(u(1))=D^{\delta}(v(1))=0
\end{array}\right.
$$

is equivalent to the integral equations

$$
u(t)=\int_{0}^{1} \mathcal{G}^{\beta}(t, s) \phi_{q}\left(\int_{0}^{s}(s-\tau)^{\sigma-1} \mathcal{F}_{1}(\tau) \psi_{1}\left(\tau, u(\tau),{ }^{c} D^{\sigma}\left(\phi_{p} D^{\beta} u(\tau)\right)\right) d \tau\right) d s
$$

and

$$
v(t)=\int_{0}^{1} \mathcal{G}^{\beta}(t, s) \phi_{q}\left(\int_{0}^{s}(s-\tau)^{\sigma-1} \mathcal{F}_{2}(\tau) \psi_{2}\left(\tau,{ }^{c} D^{\rho}\left(\phi_{p} D^{\beta} u(\tau)\right), v(\tau)\right) d \tau\right) d s
$$

where $\mathcal{G}^{\beta}(t, s)$ is Green's function given by

$$
\mathcal{G}^{\beta}(t, s)= \begin{cases}\frac{-(t-s)^{\beta-1}}{\Gamma(\beta)}-\frac{t^{\beta-1}(1-s)^{\beta-\delta-1}}{\Gamma(\beta)}, & s \leq t \leq 1, t \in \mathrm{J}=(0,1) \\ \frac{-t^{\beta-1}(1-s)^{\beta-\delta-1}}{\Gamma(\beta)}, & t \leq s \leq 1 .\end{cases}
$$

Proof Let $u, v \in \mathcal{C}(\mathrm{J}, \mathcal{X})$ be the solution of (3.1), then

$$
\left\{\begin{array}{l}
{ }^{c} D^{\sigma}\left[\phi_{p} D^{\beta} u(t)\right]+\mathcal{F}_{1}(t) \psi_{1}\left(t, u(t),{ }^{c} D^{\rho}\left[\phi_{p} D^{\beta} v(t)\right]\right)=0, \quad t \in \mathrm{J}, \\
{ }^{c} D^{\rho}\left[\phi_{p} D^{\beta} v(t)\right]+\mathcal{F}_{2}(t) \psi_{2}\left(t,{ }^{c} D^{\sigma}\left[\phi_{p} D^{\beta} u(t)\right], v(t)\right)=0, \quad t \in \mathrm{J}, \\
\left(\left[\phi_{p} D^{\beta} u(0)\right]\right)^{(j)}=0, \quad j=0,1, \ldots, m-1, \\
\left(\left[\phi_{p} D^{\beta} v(0)\right]\right)^{(j)}=0, \quad j=0,1, \ldots, m-1, \\
I^{k-\beta}(u(0))=I^{k-\beta}(v(0))=0, \quad k=2,3, \ldots, m, \\
D^{\delta}(u(1))=D^{\delta}(v(1))=0 .
\end{array}\right.
$$


Since

$$
{ }^{c} D^{\sigma}\left(\phi_{p}\left(D^{\beta} u(t)\right)+\mathcal{F}_{1}(t) \psi_{1}\left(t, u(t),{ }^{c} D^{\rho}\left[\phi_{p} D^{\beta} v(t)\right]\right)=0\right.
$$

where $m-1<\sigma<m, m-1<\beta \leq m, t \in \mathrm{J}$. Using Lemma 2.4, we have

$$
\begin{aligned}
\phi_{p}\left(D^{\beta} u(t)\right)= & -b_{0}-b_{1} t-b_{2} t^{2}-\cdots-b_{m-1} t^{m-1} \\
& -\frac{1}{\Gamma(\sigma)} \int_{0}^{t}(t-s)^{\sigma-1} \mathcal{F}_{1}(s) \psi_{1}\left(s, u(s),{ }^{c} D^{\rho}\left[\phi_{p} D^{\beta} v(s)\right]\right) .
\end{aligned}
$$

The $\left.\left(\phi_{p}\left(D^{\beta} u(t)\right)\right)^{(0)}\right|_{t=0}=0$ implies that $-b_{0}=0$ or $b_{0}=0$. Therefore (3.4) becomes

$$
\begin{aligned}
\phi_{p}\left(D^{\beta} u(t)\right)= & -b_{1} t-b_{2} t^{2}-\cdots-b_{m-1} t^{m-1} \\
& -\frac{1}{\Gamma(\sigma)} \int_{0}^{t}(t-s)^{\sigma-1} \mathcal{F}_{1}(s) \psi_{1}\left(s, u(s),{ }^{c} D^{\rho}\left[\phi_{p} D^{\beta} v(s)\right]\right) .
\end{aligned}
$$

Differentiating (3.5) with respect to $t$, we have

$$
\begin{aligned}
\left(\phi_{p}\left(D^{\beta} u(t)\right)\right)^{\prime}= & -b_{1}-2 b_{2} t-\cdots-(m-1) b_{m-1} t^{m-2} \\
& -\frac{1}{\Gamma(\sigma-1)} \int_{0}^{t}(t-s)^{\sigma-2} \mathcal{F}_{1}(s) \psi_{1}\left(s, u(s),{ }^{c} D^{\rho}\left[\phi_{p} D^{\beta} v(s)\right]\right) .
\end{aligned}
$$

Using the condition $\left.\left(\phi_{p}\left(D^{\beta} u(t)\right)\right)^{\prime}\right|_{t=0}=0$ implies that $b_{1}=0$. Similarly, by applying the conditions $\left.\left(\phi_{p}\left(D^{\beta} u(t)\right)\right)^{(j)}\right|_{t=0}=0$, we get $b_{j}=0, \forall j=2,3, \ldots m$. Therefore, (3.4) becomes

$$
\begin{aligned}
D^{\beta} u(t) & =-\phi_{p}^{-1}\left(I^{\sigma}\left(\mathcal{F}_{1}(t) \psi_{1}\left(t, u(t),{ }^{c} D^{\rho}\left(\phi_{p} D^{\beta} v(t)\right)\right)\right)\right. \\
& =-\phi_{q}\left(I^{\sigma} \mathcal{F}_{1}(t) \psi_{1}\left(t, u(t),{ }^{c} D^{\rho}\left(\phi_{p} D^{\beta} v(t)\right)\right) .\right.
\end{aligned}
$$

Applying $I^{\beta}$ to both sides and using Lemma 2.5, we have

$$
\begin{aligned}
u(t)= & -d_{1} t^{\beta-1}-d_{2} t^{\beta-2}-\cdots-d_{m} t^{\beta-m} \\
& -\frac{1}{\Gamma(\beta)} \int_{0}^{t}(t-s)^{\beta-1} \phi_{q}\left(I^{\sigma} \mathcal{F}_{1}(t) \psi_{1}\left(t, u(t),{ }^{c} D^{\rho}\left(\phi_{p} D^{\beta} v(t)\right)\right) .\right.
\end{aligned}
$$

Putting $\left.I^{k-\beta} u(t)\right|_{t=0}=0$ for $k=2,3, \ldots, m$, we obtain $d_{2}=d_{3}=\cdots=d_{m}=0$, and using $\left.D^{\delta} u(t)\right|_{t=1}=0$, we get

$$
d_{1}=-\frac{\Gamma(\beta-\delta)}{\Gamma(\beta)} \phi_{q}\left(\left.I^{\sigma} \mathcal{F}_{1}(t) \psi_{1}\left(t, u(t),{ }^{c} D^{\rho}\left(\phi_{p} D^{\beta} v(t)\right)\right)\right|_{t=1} .\right.
$$

It follows that

$$
\begin{aligned}
u(t)= & -\frac{1}{\Gamma(\beta)} \int_{0}^{t}(t-s)^{\beta-1} \phi_{q}\left(\int_{0}^{s}(s-\tau)^{\sigma-1} \mathcal{F}_{1}(\tau) \psi_{1}\left(\tau, u(\tau),{ }^{c} D^{\rho}\left(\phi_{p} D^{\beta} v(\tau)\right)\right) d \tau\right) d s \\
& -\frac{t^{\beta-1}}{\Gamma(\beta)} \int_{0}^{1}(1-s)^{\beta-\delta-1} \\
& \times \phi_{q}\left(\int_{0}^{s}(s-\tau)^{\sigma-1} \mathcal{F}_{1}(\tau) \psi_{1}\left(\tau, u(\tau),{ }^{c} D^{\rho}\left(\phi_{p} D^{\beta} v(\tau)\right)\right) d \tau\right) d s
\end{aligned}
$$




$$
=\int_{0}^{1} \mathcal{G}^{\beta}(t, s) \phi_{q}\left(\int_{0}^{s}(s-\tau)^{\sigma-1} \mathcal{F}_{1}(\tau) \psi_{1}\left(\tau, u(\tau),{ }^{c} D^{\rho}\left(\phi_{p} D^{\beta} v(\tau)\right)\right) d \tau\right) d s .
$$

In a similar manner, we may conclude that

$$
v(t)=\int_{0}^{1} \mathcal{G}^{\beta}(t, s) \phi_{q}\left(\int_{0}^{s}(s-\tau)^{\sigma-1} \mathcal{F}_{2}(\tau) \psi_{2}\left(\tau,{ }^{c} D^{\rho}\left(\phi_{p} D^{\beta} u(\tau)\right), v(\tau)\right) d \tau\right) d s,
$$

where $\mathcal{G}^{\beta}(t, s)$ is the Green's function defined above.

Lemma 3.2 ([8]) The Green's function $\mathcal{G}^{\beta}(t, s)$ satisfies the following properties:

$\left(\mathcal{P}_{1}\right) \mathcal{G}^{\beta}(t, s)>0, \forall 0<s, t<1$;

$\left(\mathcal{P}_{2}\right) \mathcal{G}^{\beta}(t, s)$ is a nondecreasing function and $\max _{t \in(0,1)} \mathcal{G}^{\beta}(t, s)=\mathcal{G}^{\beta}(1, s)$;

$\left(\mathcal{P}_{3}\right) t^{\beta-1} \max _{t \in(0,1)} \mathcal{G}^{\beta}(t, s) \leq \mathcal{G}^{\beta}(t, s)$ for $0<s, t<1$.

We introduce the following assumptions:

$\left(\mathbf{H}_{1}\right)$ The functions $\psi_{1}, \psi_{2}: \mathrm{J} \times \mathcal{X} \times \mathcal{X} \rightarrow \mathcal{X}$ are continuous, and $\forall u, v, \bar{u}, \bar{v} \in \mathcal{X}$ and $t \in \mathrm{J}$, there exist $\mathcal{M}_{\psi_{1}}, \mathcal{M}_{\psi_{2}}, \mathcal{M}^{\prime}{ }_{\psi 1}, \mathcal{M}_{\psi_{2}}^{\prime}>0$ such that

$$
\left\|\psi_{1}(t, u, v)-\psi_{1}(t, \bar{u}, \bar{v})\right\| \leq \mathcal{M}_{\psi_{1}}\|u-\bar{u}\|+\mathcal{M}_{\psi_{1}}^{\prime}\|v-\bar{v}\|
$$

and

$$
\left\|\psi_{2}(t, u, v)-\psi_{2}(t, \bar{u}, \bar{v})\right\| \leq \mathcal{M}_{\psi_{2}}\|u-\bar{u}\|+\mathcal{M}_{\psi_{2}}^{\prime}\|v-\bar{v}\| .
$$

$\left(\mathbf{H}_{2}\right)$ The functions $\psi_{1}, \psi_{2}: J \times \mathcal{X} \times \mathcal{X} \rightarrow \mathcal{X}$ are completely continuous such that, $\forall u, v \in$ $\mathcal{X}$ and $t \in \mathrm{J}$, there exist nondecreasing continuous linear functions $\mu_{\psi_{1}}, \mu_{\psi_{2}}: \mathcal{R}^{+} \rightarrow$ $\mathcal{R}$ such that

$$
\left\|\psi_{1}(t, u, v)\right\| \leq \phi_{p}\left(\mu_{\psi_{1}}\|u\|+\mu_{\psi_{1}}^{\prime}\|v\|\right)
$$

and

$$
\left\|\psi_{2}(t, u, v)\right\| \leq \phi_{p}\left(\mu_{\psi_{2}}\|u\|+\mu_{\psi_{2}}^{\prime}\|v\|\right)
$$

where

$$
\begin{aligned}
& \sup \left\{\mu_{\psi_{1}}(t), t \in \mathrm{J}\right\}=\mu_{\psi_{1}}, \quad \sup \left\{\mu_{\psi_{2}}(t), t \in \mathrm{J}\right\}=\mu_{\psi_{2}}, \\
& \sup \left\{\mu_{\psi_{1}}^{\prime}(t), t \in \mathrm{J}\right\}=\mu_{\psi_{1}}^{\prime}, \quad \sup \left\{\mu_{\psi_{2}}^{\prime}(t), t \in \mathrm{J}\right\}=\mu_{\psi_{2}}^{\prime} .
\end{aligned}
$$

$\left(\mathbf{H}_{3}\right)$ The functions $\mathcal{F}_{1}, \mathcal{F}_{2}:(0,1) \rightarrow \mathcal{X}$ are nonzero and continuous with

$$
\left\|\mathcal{F}_{1}\right\|=\max _{t \in \mathrm{J}}\left|\mathcal{F}_{1}\right|<\infty^{+}, \quad\left\|\mathcal{F}_{2}\right\|=\max _{t \in \mathrm{J}}\left|\mathcal{F}_{2}\right|<\infty^{+}
$$

Let $\mathcal{B}_{r} \subset \mathbf{B}=\mathrm{C}(\mathrm{J}, \mathcal{X}) \times \mathrm{C}(\mathrm{J}, \mathcal{X})$ be a cone of nonnegative functions of the form

$$
\mathcal{B}_{r}=\left\{(u, v) \in \mathbf{B}, \min _{t \in \mathrm{J}}(u(t)+v(t)) \geq t^{\beta}\|(u, v)\|\right\},
$$


and

$$
\Omega(r)=\left\{\|(u, v)\|<r,\|u\|<\frac{r}{2},\|v\|<\frac{r}{2}\right\}, \quad \partial \Omega(r)=\{\|(u, v)\|=r\} .
$$

Consider the operator $\mathcal{H}^{*}=\left(\mathcal{H}^{*}{ }_{1}, \mathcal{H}^{*}{ }_{2}\right): \mathcal{B}_{r} /(0,0) \rightarrow \mathbf{B}$, where $\mathcal{H}^{*}{ }_{1}, \mathcal{H}^{*}{ }_{2}$ are defined as follows:

$$
\left\{\begin{array}{l}
\mathcal{H}^{*}{ }_{1}(u, v)(t) \\
\quad=\int_{0}^{1} \mathcal{G}^{\beta}(t, s) \phi_{q}\left(\frac{1}{\sigma} \int_{0}^{s}(s-\tau)^{\sigma-1} \mathcal{F}_{1}(\tau) \psi_{1}\left(\tau, u(\tau),{ }^{c} D^{\rho}\left(\phi_{p} D^{\beta} v(\tau)\right)\right) d \tau\right) d s, \\
\mathcal{H}^{*}{ }_{2}(u, v)(t) \\
\quad=\int_{0}^{1} \mathcal{G}^{\beta}(t, s) \phi_{q}\left(\frac{1}{\rho} \int_{0}^{s}(s-\tau)^{\rho-1} \mathcal{F}_{2}(\tau) \psi_{2}\left(\tau,{ }^{c} D^{\sigma}\left(\phi_{p} D^{\beta} u(\tau)\right), v(\tau)\right) d \tau\right) d s .
\end{array}\right.
$$

Theorem 3.3 Let assumptions $\left(\mathbf{H}_{1}\right)$ to $\left(\mathbf{H}_{3}\right)$ hold. Then (1.1) has at least one solution.

Proof For any $(u, v) \in \overline{\Omega\left(r_{2}\right)} / \Omega\left(r_{1}\right)$, and using Lemma 3.2, we have

$$
\begin{aligned}
\mathcal{H}^{*}{ }_{1}(u, v)(t) \\
\quad=\int_{0}^{1} \mathcal{G}^{\beta}(t, s) \phi_{q}\left(\frac{1}{\sigma} \int_{0}^{s}(s-\tau)^{\sigma-1} \mathcal{F}_{1}(\tau) \psi_{1}\left(\tau, u(\tau),{ }^{c} D^{\rho}\left(\phi_{p} D^{\beta} v(\tau)\right)\right) d \tau\right) d s \\
\quad \leq \int_{0}^{1} \mathcal{G}^{\beta}(1, s) \phi_{q}\left(\frac{1}{\sigma} \int_{0}^{s}(s-\tau)^{\sigma-1} \mathcal{F}_{1}(\tau) \psi_{1}\left(\tau, u(\tau),{ }^{c} D^{\rho}\left(\phi_{p} D^{\beta} v(\tau)\right)\right) d \tau\right) d s
\end{aligned}
$$

and

$$
\begin{aligned}
\mathcal{H}^{*}{ }_{1}(u, v)(t) & \\
= & \int_{0}^{1} \mathcal{G}^{\beta}(t, s) \phi_{q}\left(\frac{1}{\sigma} \int_{0}^{s}(s-\tau)^{\sigma-1} \mathcal{F}_{1}(\tau) \psi_{1}\left(\tau, u(\tau),{ }^{c} D^{\rho}\left(\phi_{p} D^{\beta} \nu(\tau)\right)\right) d \tau\right) d s \\
\geq & t^{\beta-1} \int_{0}^{1} \mathcal{G}^{\beta}(1, s) \\
& \times \phi_{q}\left(\frac{1}{\sigma} \int_{0}^{s}(s-\tau)^{\sigma-1} \mathcal{F}_{1}(\tau) \psi_{1}\left(\tau, u(\tau),{ }^{c} D^{\rho}\left(\phi_{p} D^{\beta} v(\tau)\right)\right) d \tau\right) d s .
\end{aligned}
$$

By the help of inequalities (3.7) and (3.8), we have

$$
\mathcal{H}^{*}{ }_{1}(u, v)(t) \geq t^{\beta-1}\left\|\mathcal{H}^{*}{ }_{1}(u, v)(t)\right\|
$$

Similarly, we may obtain

$$
\mathcal{H}^{*}{ }_{2}(u, v)(t) \geq t^{\beta-1}\left\|\mathcal{H}^{*}{ }_{2}(u, v)(t)\right\|
$$

Combining (3.11) and (3.12), we get

$$
\mathcal{H}^{*}(u, v)(t) \geq t^{\beta-1}\left\|\mathcal{H}^{*}(u, v)(t)\right\|
$$

Thus $\mathcal{H}^{*}: \overline{\Omega\left(r_{2}\right)} / \Omega\left(r_{1}\right) \rightarrow \mathbf{B}$ is closed. 
For the uniform boundedness of the operator $\mathcal{H}^{*}$, we consider

$$
\begin{aligned}
& \left\|\mathcal{H}^{*}(u, v)(t)\right\| \\
& =\sup _{t \in \mathrm{J}}\left|\mathcal{H}^{*}(u, v)(t)\right| \\
& =\sup _{t \in \mathrm{J}}\left|\left(\mathcal{H}^{*}{ }_{1}, \mathcal{H}^{*}{ }_{2}\right)(u, v)(t)\right| \\
& \leq \sup _{t \in \mathrm{J}} \mid\left(\mathcal{H}^{*}{ }_{1}(u, v)(t)\left|+\sup _{t \in \mathrm{J}}\right| \mathcal{H}^{*}{ }_{2}(u, v)(t) \mid\right. \\
& \leq \sup _{t \in \mathrm{J}} \mid \int_{0}^{1} \mathcal{G}^{\beta}(t, s) \\
& \times \phi_{q}\left(\frac{1}{\Gamma(\sigma)} \int_{0}^{s}(s-\tau)^{\sigma-1} \mathcal{F}_{1}(\tau) \psi_{1}\left(\tau, u(\tau),{ }^{c} D^{\rho}\left(\phi_{p} D^{\beta} v(\tau)\right)\right) d \tau\right) d s \mid \\
& +\sup _{t \in \mathrm{J}} \mid \int_{0}^{1} \mathcal{G}^{\beta}(t, s) \\
& \times \phi_{q}\left(\frac{1}{\Gamma(\rho)} \int_{0}^{s}(s-\tau)^{\rho-1} \mathcal{F}_{2}(\tau) \psi_{2}\left(\tau,{ }^{c} D^{\sigma}\left(\phi_{p} D^{\beta} u(\tau)\right), v(\tau)\right) d \tau\right) d s \mid \\
& \leq \int_{0}^{1}\left|\mathcal{G}^{\beta}(1, s)\right| \\
& \times \phi_{q}\left(\frac{1}{\Gamma(\sigma)} \int_{0}^{s}(s-\tau)^{\sigma-1}\left\|\mathcal{F}_{1}(\tau)\right\|\left\|\psi_{1}\left(\tau, u(\tau),{ }^{c} D^{\rho}\left(\phi_{p} D^{\beta} v(\tau)\right)\right)\right\| d \tau\right) d s \\
& +\int_{0}^{1}\left|\mathcal{G}^{\beta}(1, s)\right| \\
& \times \phi_{q}\left(\frac{1}{\Gamma(\rho)} \int_{0}^{s}(s-\tau)^{\rho-1}\left\|\mathcal{F}_{2}(\tau)\right\|\left\|\psi_{2}\left(\tau,{ }^{c} D^{\sigma}\left(\phi_{p} D^{\beta} u(\tau), v(\tau)\right)\right)\right\| d \tau\right) d s \\
& \leq\left(\frac{1}{\Gamma(\beta+1)}-\frac{1}{\Gamma(\beta-\delta) \Gamma(\beta)}\right)\left[\frac{1}{\Gamma(\sigma+1)}\right]^{q-1} \\
& \times\left\|\mathcal{F}_{1}\right\|^{q-1}\left(\frac{\mu_{\psi_{1}}\|u\|+\mu_{\psi_{1}}^{\prime} \mu_{\psi_{2}}^{\prime}\left\|\mathcal{F}_{2}\right\|\|v\|}{1-\left\|\mathcal{F}_{1}\right\|\left\|\mathcal{F}_{2}\right\| \mu_{\psi_{1}}^{\prime} \mu_{\psi_{2}}}\right) \\
& +\left(\frac{1}{\Gamma(\beta+1)}-\frac{1}{\Gamma(\beta-\delta) \Gamma(\beta)}\right)\left[\frac{1}{\Gamma(\rho+1)}\right]^{q-1} \\
& \times\left\|\mathcal{F}_{2}\right\|^{q-1}\left(\frac{\mu_{\psi_{1}} \mu_{\psi_{2}}\left\|\mathcal{F}_{1}\right\|\|u\|+\mu_{\psi_{2}}\|v\|}{1-\left\|\mathcal{F}_{1}\right\|\left\|\mathcal{F}_{2}\right\| \mu_{\psi_{1}}^{\prime} \mu_{\psi_{2}}}\right) \\
& =\left(\frac{1}{\Gamma(\beta+1)}-\frac{1}{\Gamma(\beta-\delta) \Gamma(\beta)}\right)\left[\left(\frac{1}{\Gamma(\sigma+1)}\right)^{q-1}\right. \\
& \times\left\|\mathcal{F}_{1}\right\|^{q-1}\left(\frac{\mu_{\psi_{1}}\|u\|+\mu_{\psi_{1}} \mu_{\psi_{2}}^{\prime}\left\|\mathcal{F}_{2}\right\|\|v\|}{1-\left\|\mathcal{F}_{1}\right\|\left\|\mathcal{F}_{2}\right\| \mu_{\psi_{1}}^{\prime} \mu_{\psi_{2}}}\right) \\
& \left.+\left(\frac{1}{\Gamma(\rho+1)}\right)^{q-1}\left\|\mathcal{F}_{2}\right\|^{q-1}\left(\frac{\mu_{\psi_{1}} \mu_{\psi_{2}}\left\|\mathcal{F}_{1}\right\|\|u\|+\mu_{\psi_{2}}^{\prime}\|v\|}{1-\left\|\mathcal{F}_{1}\right\|\left\|\mathcal{F}_{2}\right\| \mu_{\psi_{1}}^{\prime} \mu_{\psi_{2}}}\right)\right] \\
& <\infty \text {. }
\end{aligned}
$$

Hence $\mathcal{H}^{*}$ is a uniformly bounded operator. 
Now, we show that the operator $\mathcal{H}^{*}$ is continuous and compact. For this reason, we construct a sequence $\xi_{n}=\left(u_{n}, v_{n}\right)$ such that $\left(u_{n}, v_{n}\right) \rightarrow(u, v)$ as $n \rightarrow \infty$. Therefore, we have

$$
\begin{aligned}
& \left\|\left(\mathcal{H}^{*}\left(u_{n}, v_{n}\right)-\mathcal{H}^{*}(u, v)\right)\right\| \\
& \left.=\|\left(\mathcal{H}^{*}{ }_{1}, \mathcal{H}^{*}{ }_{2}\right)\left(u_{n}, v_{n}\right)-\left(\mathcal{H}^{*}{ }_{1}, \mathcal{H}^{*}{ }_{2}\right)(u, v)\right) \| \\
& \leq\left\|\left(\mathcal{H}^{*}{ }_{1}\left(u_{n}, v_{n}\right)-\mathcal{H}^{*}{ }_{1}(u, v)\right)\right\|+\left\|\left(\mathcal{H}^{*}{ }_{2}\left(u_{n}, v_{n}\right)-\mathcal{H}^{*}{ }_{2}(u, v)\right)\right\| \\
& =\sup _{t \in \mathrm{J}} \mid \int_{0}^{1} \mathcal{G}^{\beta}(t, s) \\
& \times \phi_{q}\left(\frac{1}{\Gamma(\sigma)} \int_{0}^{s}(s-\tau)^{\sigma-1} \mathcal{F}_{1}(\tau) \psi_{1}\left(\tau, u_{n}(\tau),{ }^{c} D^{\rho}\left(\phi_{p} D^{\beta} v_{n}(\tau)\right)\right) d \tau\right) d s \\
& -\int_{0}^{1} \mathcal{G}^{\beta}(t, s) \phi_{q}\left(\frac{1}{\Gamma(\sigma)} \int_{0}^{s}(s-\tau)^{\sigma-1} \mathcal{F}_{1}(\tau) \psi_{1}\left(\tau, u(\tau),{ }^{c} D^{\rho}\left(\phi_{p} D^{\beta} v(\tau)\right)\right) d \tau\right) d s \\
& +\sup _{t \in \mathrm{J}} \mid \int_{0}^{1} \mathcal{G}^{\beta}(t, s) \\
& \times \phi_{q}\left(\frac{1}{\Gamma(\rho)} \int_{0}^{s}(s-\tau)^{\rho-1} \mathcal{F}_{2}(\tau) \psi_{2}\left(\tau,{ }^{c} D^{\sigma}\left(\phi_{p} D^{\beta} u_{n}(\tau)\right), v_{n}(\tau)\right) d \tau\right) d s \\
& -\int_{0}^{1} \mathcal{G}^{\beta}(t, s) \phi_{q}\left(\frac{1}{\Gamma(\rho)} \int_{0}^{s}(s-\tau)^{\rho-1} \mathcal{F}_{2}(\tau) \psi_{2}\left(\tau,{ }^{c} D^{\sigma}\left(\phi_{p} D^{\beta} u(\tau)\right), v(\tau)\right) d \tau\right) d s \\
& \leq \int_{0}^{1}\left|\mathcal{G}^{\beta}(t, s)\right| \\
& \times\left\{\| \phi_{q}\left(\frac{1}{\Gamma(\sigma)} \int_{0}^{s}(s-\tau)^{\sigma-1} \mathcal{F}_{1}(\tau) \psi_{1}\left(\tau, u_{n}(\tau),{ }^{c} D^{\rho}\left(\phi_{p} D^{\beta} v_{n}(\tau)\right)\right) d \tau\right) d s\right. \\
& \left.-\phi_{q}\left(\frac{1}{\Gamma(\sigma)} \int_{0}^{s}(s-\tau)^{\sigma-1} \mathcal{F}_{1}(\tau) \psi_{1}\left(\tau, u(\tau),{ }^{c} D^{\rho}\left(\phi_{p} D^{\beta} v(\tau)\right)\right) d \tau\right) d s \|\right\} \\
& +\int_{0}^{1}\left|\mathcal{G}^{\beta}(t, s)\right| \\
& \times\left\{\| \phi_{q}\left(\frac{1}{\Gamma(\rho)} \int_{0}^{s}(s-\tau)^{\rho-1} \mathcal{F}_{2}(\tau) \psi_{2}\left(\tau,{ }^{c} D^{\sigma}\left(\phi_{p} D^{\beta} u_{n}(\tau)\right), v_{n}(\tau)\right) d \tau\right) d s\right. \\
& \left.-\phi_{q}\left(\frac{1}{\Gamma(\rho)} \int_{0}^{s}(s-\tau)^{\rho-1} \mathcal{F}_{2}(\tau) \psi_{2}\left(\tau,{ }^{c} D^{\sigma}\left(\phi_{p} D^{\beta} u(\tau)\right), v(\tau)\right) d \tau\right) d s \|\right\} \\
& \leq(q-1) \varrho^{2} \int_{0}^{1}\left|\mathcal{G}^{\beta}(t, s)\right| \\
& \times\left\{\frac{1}{\Gamma(\sigma)} \int_{0}^{s}(s-\tau)^{\sigma-1}\left\|\mathcal{F}_{1}(\tau)\right\| \| \psi_{1}\left(\tau, u_{n}(\tau),{ }^{c} D^{\rho}\left(\phi_{p} D^{\beta} v_{n}(\tau)\right)\right)\right. \\
& -\psi_{1}\left(\tau, u(\tau),{ }^{c} D^{\rho}\left(\phi_{p} D^{\beta} v(\tau)\right) \| d \tau\right) d s \\
& +\frac{1}{\Gamma(\rho)} \int_{0}^{s}(s-\tau)^{\rho-1}\left\|\mathcal{F}_{2}(\tau)\right\| \| \psi_{2}\left(\tau,{ }^{c} D^{\sigma}\left(\phi_{p} D^{\beta} u_{n}(\tau)\right), v_{n}(\tau)\right) \\
& \left.-\psi_{2}\left(\tau,{ }^{c} D^{\sigma}\left(\phi_{p} D^{\beta} u(\tau), v(\tau)\right) \| d \tau\right) d s\right\} \\
& \leq(q-1) \varrho^{2} \int_{0}^{1}\left|\mathcal{G}^{\beta}(t, s)\right|\left\{\frac{\mathcal{M}_{\psi_{1}}\left\|\mathcal{F}_{1}\right\|\left\|u_{n}-u\right\|+\mathcal{M}_{\psi_{1}}^{\prime} \mathcal{M}_{\psi_{2}}^{\prime}\left\|\mathcal{F}_{1}\right\|\left\|\mathcal{F}_{2}\right\|\left\|v_{n}-v\right\|}{\Gamma(\sigma+1)}\right.
\end{aligned}
$$




$$
\left.+\frac{\mathcal{M}_{\psi_{1}} \mathcal{M}_{\psi_{2}}\left\|\mathcal{F}_{1}\right\| \mathcal{F}_{2}\|\| u_{n}-u\left\|+\mathcal{M}_{\psi_{2}}^{\prime}\right\| \mathcal{F}_{2}\|\| v_{n}-v \|}{\Gamma(\rho+1)}\right\}
$$

$\rightarrow 0, \quad$ as $n \rightarrow \infty$.

Therefore, $\left\|\mathcal{H}^{*}\left(u_{n}, v_{n}\right)-\mathcal{H}^{*}(u, v)\right\| \rightarrow 0$ as $n \rightarrow \infty$. Hence $\mathcal{H}^{*}$ is continuous.

For equicontinuity, take $v_{1}, v_{2} \in \mathrm{J}$ with $v_{1}<v_{2}$, and for any $(u, v) \in \Omega(r)$, we have

$$
\begin{aligned}
& \left\|\left(\mathcal{H}^{*}(u, v)\left(v_{1}\right)-\mathcal{H}^{*}(u, v)\left(v_{2}\right)\right)\right\| \\
& \leq\left\|\left(\left(\mathcal{H}^{*}{ }_{1}(u, v)\left(v_{1}\right)-\mathcal{H}^{*}{ }_{1}(u, v)\left(v_{2}\right)\right)\right)\right\|+\left\|\left(\mathcal{H}^{*}{ }_{2}(u, v)\left(v_{1}\right)-\mathcal{H}^{*}{ }_{2}(u, v)\left(v_{2}\right)\right)\right\| \\
& =\sup _{t \in \mathrm{J}} \mid \int_{0}^{1} \mathcal{G}^{\beta}\left(v_{1}, s\right) \phi_{q}\left(\frac{1}{\Gamma(\sigma)} \int_{0}^{s}(s-\tau)^{\sigma-1} \mathcal{F}_{1}(\tau) \psi_{1}\left(\tau, u(\tau),{ }^{c} D^{\rho}\left(\phi_{p} D^{\beta} v(\tau)\right)\right) d \tau\right) d s \\
& -\int_{0}^{1} \mathcal{G}^{\beta}\left(v_{2}, s\right) \phi_{q}\left(\frac{1}{\Gamma(\sigma)} \int_{0}^{s}(s-\tau)^{\sigma-1} \mathcal{F}_{1}(\tau) \psi_{1}\left(\tau, u(\tau),{ }^{c} D^{\rho}\left(\phi_{p} D^{\beta} v(\tau)\right)\right) d \tau\right) d s \mid \\
& +\sup _{t \in \mathrm{J}} \mid \int_{0}^{1} \mathcal{G}^{\beta}\left(\tau_{1}, s\right) \\
& \times \phi_{q}\left(\frac{1}{\Gamma(\rho)} \int_{0}^{s}(s-\tau)^{\rho-1} \mathcal{F}_{2}(\tau) \psi_{1}\left(\tau,{ }^{c} D^{\sigma}\left(\phi_{p} D^{\beta} u(\tau), v(\tau)\right)\right) d \tau\right) d s \\
& -\int_{0}^{1} \mathcal{G}^{\beta}\left(v_{2}, s\right) \phi_{q}\left(\frac{1}{\Gamma(\rho)} \int_{0}^{s}(s-\tau)^{\rho-1} \mathcal{F}_{2}(\tau) \psi_{1}\left(\tau,{ }^{c} D^{\sigma}\left(\phi_{p} D^{\beta} u(\tau), v(\tau)\right)\right) d \tau\right) d s \\
& \leq \int_{0}^{1}\left|\mathcal{G}^{\beta}\left(\tau_{1}, s\right)-\mathcal{G}^{\beta}\left(\tau_{2}, s\right)\right| \\
& \times \phi_{q}\left(\frac{1}{\Gamma(\sigma)} \int_{0}^{s}(s-\tau)^{\sigma-1}\left\|\mathcal{F}_{1}\right\|\left\|\psi_{1}\left(\tau, u(\tau),{ }^{c} D^{\rho}\left(\phi_{p} D^{\beta} v(\tau)\right)\right)\right\| d \tau\right) d s \\
& +\int_{0}^{1}\left|\mathcal{G}^{\beta}\left(\tau_{1}, s\right)-\mathcal{G}^{\beta}\left(\tau_{2}, s\right)\right| \\
& \times \phi_{q}\left(\frac{1}{\Gamma(\rho)} \int_{0}^{s}(s-\tau)^{\rho-1}\left\|\mathcal{F}_{2}\right\|\left\|\psi_{1}\left(\tau,{ }^{c} D^{\sigma}\left(\phi_{p} D^{\beta} u(\tau), v(\tau)\right)\right)\right\| d \tau\right) d s \\
& \leq\left(\frac{\left|v_{1}^{\beta}-v_{2}^{\beta}\right|}{\Gamma(\beta+1)}+\frac{\left|v_{1}^{\beta-1}-v_{2}^{\beta-1}\right|}{\Gamma(\beta-\delta) \Gamma(\beta+1)}\right)\left[\frac{1}{\Gamma(\sigma+1)}\right]^{q-1} \\
& \times\left\|\mathcal{F}_{1}\right\|^{q-1}\left(\frac{\mu_{\psi_{1}}\|u\|+\mu_{\psi_{1}}^{\prime} \mu_{\psi_{2}}^{\prime}\left\|\mathcal{F}_{2}\right\|\|v\|}{1-\left\|\mathcal{F}_{1}\right\|\left\|\mathcal{F}_{2}\right\| \mu_{\psi_{1}}^{\prime} \mu_{\psi_{2}}}\right) \\
& \leq\left(\frac{\left|v_{1}^{\beta}-v_{2}^{\beta}\right|}{\Gamma(\beta+1)}+\frac{\left|v_{1}^{\beta-1}-v_{2}^{\beta-1}\right|}{\Gamma(\beta-\delta) \Gamma(\beta+1)}\right)\left[\frac{1}{\Gamma(\rho+1)}\right]^{q-1} \\
& \times\left\|\mathcal{F}_{2}\right\|^{q-1}\left(\frac{\mu_{\psi_{1}} \mu_{\psi_{2}}\|u\|+\mu_{\psi_{2}}^{\prime}\|v\|}{1-\left\|\mathcal{F}_{1}\right\|\left\|\mathcal{F}_{2}\right\| \mu_{\psi_{1}}^{\prime} \mu_{\psi_{2}}}\right) .
\end{aligned}
$$

This implies that $\left\|\mathcal{H}^{*}(u, v)\left(v_{1}\right)-\mathcal{H}^{*}(u, v)\left(v_{2}\right)\right\| \rightarrow 0$ as $v_{1} \rightarrow v_{2}$. Therefore $\mathcal{H}^{*}$ is relatively compact. By Arzelä-Ascolli theorem, $\mathcal{H}^{*}$ is compact and hence completely continuous operator.

Now let us define a set

$$
\left.\mathrm{W}=\left\{(u, v) \in \overline{\Omega\left(r_{2}\right)} / \Omega\left(r_{1}\right)\right) \text { there exist } \lambda \in[0,1] \text { such that }(u, v)=\lambda \mathcal{H}(u, v)\right\} \text {. }
$$


We will show that $\mathrm{W}$ is bounded. Suppose on the contrary that $\mathrm{W}$ is unbounded. Let $(u, v) \in \mathrm{W}$ such that $\|(u, v)\|=\mathcal{K} \rightarrow \infty$. But

$$
\begin{aligned}
\|(u, v)\|= & \|\lambda \mathcal{H}(u, v)\| \\
\leq & \|\mathcal{H}(u, v)\| \\
\leq & \left(\frac{1}{\Gamma(\beta+1)}-\frac{1}{\Gamma(\beta-\delta) \Gamma(\beta)}\right) \\
& \times\left[\left(\frac{1}{\Gamma(\sigma+1)}\right)^{q-1}\left\|\mathcal{F}_{1}\right\|^{q-1}\left(\frac{\mu_{\psi_{1}}\|u\|+\mu_{\psi_{1}} \mu_{\psi_{2}}^{\prime}\left\|\mathcal{F}_{2}\right\|\|v\|}{1-\left\|\mathcal{F}_{1}\right\|\left\|\mathcal{F}_{2}\right\| \mu_{\psi_{1}}^{\prime} \mu_{\psi_{2}}}\right)\right. \\
& \left.+\left(\frac{1}{\Gamma(\rho+1)}\right)^{q-1}\left\|\mathcal{F}_{2}\right\|^{q-1}\left(\frac{\mu_{\psi_{1}} \mu_{\psi_{2}}\left\|\mathcal{F}_{1}\right\|\|u\|+\mu_{\psi_{2}}^{\prime}\|v\|}{1-\left\|\mathcal{F}_{1}\right\|\left\|\mathcal{F}_{2}\right\| \mu_{\psi_{1}}^{\prime} \mu_{\psi_{2}}}\right)\right] .
\end{aligned}
$$

This implies that

$$
\begin{aligned}
\|(u, v)\| \leq & \left(\frac{1}{\Gamma(\beta+1)}-\frac{1}{\Gamma(\beta-\delta) \Gamma(\beta)}\right) \\
& \times\left[\left(\frac{1}{\Gamma(\sigma+1)}\right)^{q-1}\left\|\mathcal{F}_{1}\right\|^{q-1}\left(\frac{\mu_{\psi_{1}}\|u\|+\mu_{\psi_{1}} \mu_{\psi_{2}}^{\prime}\left\|\mathcal{F}_{2}\right\|\|v\|}{1-\left\|\mathcal{F}_{1}\right\|\left\|\mathcal{F}_{2}\right\| \mu_{\psi_{1}}^{\prime} \mu_{\psi_{2}}}\right)\right. \\
& \left.+\left(\frac{1}{\Gamma(\rho+1)}\right)^{q-1}\left\|\mathcal{F}_{2}\right\|^{q-1}\left(\frac{\mu_{\psi_{1}} \mu_{\psi_{2}}\left\|\mathcal{F}_{1}\right\|\|u\|+\mu_{\psi_{2}}^{\prime}\|v\|}{1-\left\|\mathcal{F}_{1}\right\|\left\|\mathcal{F}_{2}\right\| \mu_{\psi_{1}}^{\prime} \mu_{\psi_{2}}}\right)\right]
\end{aligned}
$$

equivalently

$$
\begin{aligned}
1 \leq & \frac{1}{\|(u, v)\|}\left(\frac{1}{\Gamma(\beta+1)}-\frac{1}{\Gamma(\beta-\delta) \Gamma(\beta)}\right) \\
& \times\left[\left(\frac{1}{\Gamma(\sigma+1)}\right)^{q-1}\left\|\mathcal{F}_{1}\right\|^{q-1}\left(\frac{\mu_{\psi_{1}}\|u\|+\mu_{\psi_{1}} \mu_{\psi_{2}}^{\prime}\left\|\mathcal{F}_{2}\right\|\|v\|}{1-\left\|\mathcal{F}_{1}\right\|\left\|\mathcal{F}_{2}\right\| \mu_{\psi_{1}}^{\prime} \mu_{\psi_{2}}}\right)\right. \\
& \left.+\left(\frac{1}{\Gamma(\rho+1)}\right)^{q-1}\left\|\mathcal{F}_{2}\right\|^{q-1}\left(\frac{\mu_{\psi_{1}} \mu_{\psi_{2}}\left\|\mathcal{F}_{1}\right\|\|u\|+\mu_{\psi_{2}}^{\prime}\|v\|}{1-\left\|\mathcal{F}_{1}\right\|\left\|\mathcal{F}_{2}\right\| \mu_{\psi_{1}}^{\prime} \mu_{\psi_{2}}}\right)\right] \\
= & \frac{1}{\mathcal{K}}\left(\frac{1}{\Gamma(\beta+1)}-\frac{1}{\Gamma(\beta-\delta) \Gamma(\beta)}\right) \\
& \times\left[\left(\frac{1}{\Gamma(\sigma+1)}\right)^{q-1}\left\|\mathcal{F}_{1}\right\|^{q-1}\left(\frac{\mu_{\psi_{1}}\|u\|+\mu_{\psi_{1}} \mu_{\psi_{2}}^{\prime}\left\|\mathcal{F}_{2}\right\|\|v\|}{1-\left\|\mathcal{F}_{1}\right\|\left\|\mathcal{F}_{2}\right\| \mu_{\psi_{1}}^{\prime} \mu_{\psi_{2}}}\right)\right. \\
& \left.+\left(\frac{1}{\Gamma(\rho+1)}\right)^{q-1}\left\|\mathcal{F}_{2}\right\|^{q-1}\left(\frac{\mu_{\psi_{1}} \mu_{\psi_{2}}\left\|\mathcal{F}_{1}\right\|\|u\|+\mu_{\psi_{2}}^{\prime}\|v\|}{1-\left\|\mathcal{F}_{1}\right\|\left\|\mathcal{F}_{2}\right\| \mu_{\psi_{1}}^{\prime} \mu_{\psi_{2}}}\right)\right] \\
\rightarrow & 0 \quad \text { as } \mathcal{K} \rightarrow \infty .
\end{aligned}
$$

This is a contradiction. Ultimately W is bounded, therefore by Lemma 2.7 the operator $\mathcal{H}$ has at least one fixed point in $\Omega\left(r_{2}\right) / \Omega\left(r_{1}\right)$, which is a solution of coupled system (1.1).

Thus, by Lemma 2.9, (1.1) has at least one solution. 
To control the growth bound of the nonlinearity functions $\psi_{1}, \psi_{2}$ and proceed to the next result, we need the following height functions. Let

$$
\left\{\begin{array}{l}
\Im_{\max _{t \in J, x>0}}(t, x)=\max \left\{\left\{\psi_{1}, \psi_{2}\right\}: t^{\beta-1} x \leq(u, v) \leq x\right\}, \\
\Im_{\min _{t \in J, x>0}}(t, x)=\min \left\{\left\{\psi_{1}, \psi_{2}\right\}: t^{\beta-1} x \leq(u, v) \leq x\right\} .
\end{array}\right.
$$

Theorem 3.4 Let assumptions $\left(\mathbf{H}_{1}\right)$ to $\left(\mathbf{H}_{3}\right)$ hold, and there exist $r^{*}, \hbar \in \mathcal{R}^{+}$such that one of the following conditions is satisfied:

$\left(\Im_{1}\right)$

$$
\hbar \leq \int_{0}^{1} \mathcal{G}^{\beta}(1, s) \phi_{q}\left(\frac{1}{\Gamma(\sigma)} \int_{0}^{s}(s-\tau)^{\sigma-1} \mathcal{F}_{1}(\tau) \Im_{\min }\left(\tau, \hbar,{ }^{c} D^{\rho}\left(\phi_{p} D^{\beta} v(\tau)\right)\right) d \tau\right) d s<\infty^{+}
$$

and

$$
\int_{0}^{1} \mathcal{G}^{\beta}(1, s) \phi_{q}\left(\frac{1}{\Gamma(\sigma)} \int_{0}^{s}(s-\tau)^{\sigma-1} \mathcal{F}_{1}(\tau) \mathfrak{I}_{\max }\left(\tau, r^{*},{ }^{c} D^{\rho}\left(\phi_{p} D^{\beta} v(\tau)\right)\right) d \tau\right) d s \leq r^{*}
$$

$\left(\Im_{2}\right)$

$$
\int_{0}^{1} \mathcal{G}^{\beta}(1, s) \phi_{q}\left(\frac{1}{\Gamma(\sigma)} \int_{0}^{s}(s-\tau)^{\sigma-1} \mathcal{F}_{1}(\tau) \mathfrak{I}_{\max }\left(\tau, \hbar,{ }^{c} D^{\rho}\left(\phi_{p} D^{\beta} v(\tau)\right)\right) d \tau\right) d s<\hbar ;
$$

and

$$
r^{*} \leq \int_{0}^{1} \mathcal{G}^{\beta}(1, s) \phi_{q}\left(\frac{1}{\Gamma(\sigma)} \int_{0}^{s}(s-\tau)^{\sigma-1} \mathcal{F}_{1}(\tau) \mathfrak{I}_{\min }\left(\tau, r^{*},{ }^{c} D^{\rho}\left(\phi_{p} D^{\beta} v(\tau)\right)\right) d \tau\right) d s<\infty^{+}
$$

$\left(\Im_{3}\right)$

$\hbar \leq \int_{0}^{1} \mathcal{G}^{\beta}(1, s) \phi_{q}\left(\frac{1}{\Gamma(\sigma)} \int_{0}^{s}(s-\tau)^{\sigma-1} \mathcal{F}_{2}(\tau) \mathfrak{I}_{\min }\left(\tau,{ }^{c} D^{\rho}\left(\phi_{p} D^{\beta} u(\tau), \hbar\right)\right) d \tau\right) d s<\infty^{+} ;$

and

$$
\int_{0}^{1} \mathcal{G}^{\beta}(1, s) \phi_{q}\left(\frac{1}{\Gamma(\sigma)} \int_{0}^{s}(s-\tau)^{\sigma-1} \mathcal{F}_{2}(\tau) \Im_{\max }\left(\tau,{ }^{c} D^{\rho}\left(\phi_{p} D^{\beta} u(\tau)\right), r^{*}\right) d \tau\right) d s \leq r^{*}
$$

$\left(\Im_{4}\right)$

$$
\int_{0}^{1} \mathcal{G}^{\beta}(1, s) \phi_{q}\left(\frac{1}{\Gamma(\sigma)} \int_{0}^{s}(s-\tau)^{\sigma-1} \mathcal{F}_{2}(\tau) \mathfrak{I}_{\max }\left(\tau,{ }^{c} D^{\rho}\left(\phi_{p} D^{\beta} u(\tau)\right), \hbar\right) d \tau\right) d s<\hbar
$$

and

$$
r^{*} \leq \int_{0}^{1} \mathcal{G}^{\beta}(1, s) \phi_{q}\left(\frac{1}{\Gamma(\sigma)} \int_{0}^{s}(s-\tau)^{\sigma-1} \mathcal{F}_{2}(\tau) \mathfrak{I}_{\min }\left(\tau,{ }^{c} D^{\rho}\left(\phi_{p} D^{\beta} u(\tau)\right), r^{*}\right) d \tau\right) d s<\infty^{+}
$$

Then problem (1.1) has a nonnegative solution $\left(u^{*}, v^{*}\right) \in \mathcal{B}_{r} \times \mathcal{B}_{r}$, so that $\hbar \leq\left\|\left(u^{*}, v^{*}\right)\right\| \leq r^{*}$. 
Proof Without loss of generality we take only $\left(\Im_{1}\right)$ and $\left(\Im_{2}\right)$. If $(u, v) \in \partial \Omega(\hbar)$, then $\|(u, v)\|=\hbar$ and $t^{\beta-1} \hbar \leq(u, v) \leq \hbar, t \in \mathrm{J}$. By (3.13) we have

$$
\begin{aligned}
&\left\|\mathcal{H}^{*}(u, v)(t)\right\| \\
&=\left\|\left(\mathcal{H}_{1}^{*}, \mathcal{H}_{2}^{*}\right)(u, v)(t)\right\| \\
&=\sup _{t \in \mathrm{J}} \int_{0}^{1} \mathcal{G}^{\beta}(t, s) \phi_{q}\left(\frac{1}{\Gamma(\sigma)} \int_{0}^{s}(s-\tau)^{\sigma-1} \mathcal{F}_{1}(\tau) \psi_{1}\left(\tau, u(\tau),{ }^{c} D^{\rho}\left(\phi_{p} D^{\beta} v(\tau)\right)\right) d \tau\right) d s \\
& \quad+\sup _{t \in \mathrm{J}} \int_{0}^{1} \mathcal{G}^{\beta}(t, s) \phi_{q}\left(\frac{1}{\Gamma(\rho)} \int_{0}^{s}(s-\tau)^{\rho-1} \mathcal{F}_{2}(\tau) \psi_{2}\left(\tau,{ }^{c} D^{\sigma}\left(\phi_{p} D^{\beta} u(\tau)\right), v(\tau)\right) d \tau\right) d s \\
& \geq t^{\beta-1} \int_{0}^{1} \mathcal{G}^{\beta}(1, s) \phi_{q}\left(\frac{1}{\Gamma(\sigma)} \int_{0}^{s}(s-\tau)^{\sigma-1} \mathcal{F}_{1}(\tau) \psi_{1}\left(\tau, u(\tau),{ }^{c} D^{\rho}\left(\phi_{p} D^{\beta} v(\tau)\right)\right) d \tau\right) d s \\
& \quad+t^{\beta-1} \int_{0}^{1} \mathcal{G}^{\beta}(1, s) \\
& \quad \times \phi_{q}\left(\frac{1}{\Gamma(\rho)} \int_{0}^{s}(s-\tau)^{\rho-1} \mathcal{F}_{2}(\tau) \psi_{2}\left(\tau,{ }^{c} D^{\sigma}\left(\phi_{p} D^{\beta} u(\tau)\right), v(\tau)\right) d \tau\right) d s \\
& \geq \int_{0}^{1} \mathcal{G}^{\beta}(1, s) \phi_{q}\left(\frac{1}{\Gamma(\sigma)} \int_{0}^{s}(s-\tau)^{\sigma-1}\left[\mathcal{F}_{1}(\tau) \mathfrak{I}_{\min _{t \in \mathrm{J}}}\left(\tau, \hbar,{ }^{c} D^{\rho}\left(\phi_{p} D^{\beta} v(\tau)\right)\right)\right] d \tau\right) d s \\
& \quad+\int_{0}^{1} \mathcal{G}^{\beta}(1, s) \phi_{q}\left(\frac{1}{\Gamma(\rho)} \int_{0}^{s}(s-\tau)^{\rho-1}\left[\mathcal{F}_{2}(\tau) \mathfrak{I}_{\min _{t \in \mathrm{J}}}\left(\tau,{ }^{c} D^{\sigma}\left(\phi_{p} D^{\beta} u(\tau)\right), \hbar\right)\right] d \tau\right) d s \\
& \geq \frac{\hbar}{2}+\frac{\hbar}{2}=\hbar=\|(u, v)\| .
\end{aligned}
$$

Thus

$$
\left\|\mathcal{H}^{*}(u, v)(t)\right\| \geq \hbar=\|(u, v)\| .
$$

When $(u, v) \in \partial \Omega\left(r^{*}\right)$, then $\|(u, v)\|=r^{*}$, and by (3.13), $t^{\beta-1} r^{*} \leq(u, v) \leq r^{*}$, we have $\Im_{\max _{t \in \mathrm{J}}} \geq\left\{\psi_{1}, \psi_{2}\right\}$, therefore

$$
\begin{aligned}
\left\|\mathcal{H}^{*}(u, v)(t)\right\| \\
=\left\|\left(\mathcal{H}_{1}^{*}, \mathcal{H}_{2}^{*}\right)(u, v)(t)\right\| \\
=\max _{t \in J} \int_{0}^{1} \mathcal{G}^{\beta}(t, s) \phi_{q}\left(\frac{1}{\Gamma(\sigma)} \int_{0}^{s}(s-\tau)^{\sigma-1} \mathcal{F}_{1}(\tau) \psi_{1}\left(\tau, u(\tau),{ }^{c} D^{\rho}\left(\phi_{p} D^{\beta} v(\tau)\right)\right) d \tau\right) d s \\
\quad+\max _{t \in J} \int_{0}^{1} \mathcal{G}^{\beta}(t, s) \\
\quad \times \phi_{q}\left(\frac{1}{\Gamma(\rho)} \int_{0}^{s}(s-\tau)^{\rho-1} \mathcal{F}_{2}(\tau) \psi_{2}\left(\tau,{ }^{c} D^{\sigma}\left(\phi_{p} D^{\beta} u(\tau)\right), v(\tau)\right) d \tau\right) d s \\
\leq t^{\beta-1} \int_{0}^{1} \mathcal{G}^{\beta}(1, s) \phi_{q}\left(\frac{1}{\Gamma(\sigma)} \int_{0}^{s}(s-\tau)^{\sigma-1} \mathcal{F}_{1}(\tau) \psi_{1}\left(\tau, u(\tau),{ }^{c} D^{\rho}\left(\phi_{p} D^{\beta} v(\tau)\right)\right) d \tau\right) d s \\
\quad+t^{\beta-1} \int_{0}^{1} \mathcal{G}^{\beta}(1, s) \\
\quad \times \phi_{q}\left(\frac{1}{\Gamma(\rho)} \int_{0}^{s}(s-\tau)^{\rho-1} \mathcal{F}_{2}(\tau) \psi_{2}\left(\tau,{ }^{c} D^{\sigma}\left(\phi_{p} D^{\beta} u(\tau)\right), v(\tau)\right) d \tau\right) d s
\end{aligned}
$$




$$
\begin{aligned}
\leq & \int_{0}^{1} \mathcal{G}^{\beta}(1, s) \phi_{q}\left(\frac{1}{\Gamma(\sigma)} \int_{0}^{s}(s-\tau)^{\sigma-1}\left[\mathcal{F}_{1}(\tau) \mathfrak{I}_{\max _{t \in \mathrm{J}}}\left(\tau, r^{*},{ }^{c} D^{\rho}\left(\phi_{p} D^{\beta} v(\tau)\right)\right)\right] d \tau\right) d s \\
& +\int_{0}^{1} \mathcal{G}^{\beta}(1, s) \\
& \times \phi_{q}\left(\frac{1}{\Gamma(\rho)} \int_{0}^{s}(s-\tau)^{\rho-1}\left[\mathcal{F}_{2}(\tau) \Im_{\max _{t \in \mathrm{J}}}\left(\tau,{ }^{c} D^{\sigma}\left(\phi_{p} D^{\beta} u(\tau)\right), r^{*}\right)\right] d \tau\right) d s \\
\leq & \frac{r^{*}}{2}+\frac{r^{*}}{2}=r^{*}=\|(u, v)\| .
\end{aligned}
$$

Thus

$$
\left\|\mathcal{H}^{*}(u, v)(t)\right\| \geq \hbar=\|(u, v)\| .
$$

Combining these inequalities, we say that $\mathcal{H}^{*}$ has a fixed point in the interval $\left[\hbar, r^{*}\right]$, say $\left(u^{*}, v^{*}\right) \in \overline{\Omega\left(r^{*}\right)} / \Omega(\hbar)$, such that $\hbar \leq\left\|\left(u^{*}, v^{*}\right)\right\| \leq r^{*}$. Next we show that $\left(u^{*}, v^{*}\right)$ is a nonnegative solution for $t \in \mathrm{J}$ as

$$
\begin{aligned}
u^{*}(t)= & \int_{0}^{1} \mathcal{G}^{\beta}(t, s) \phi_{q}\left(\frac{1}{\Gamma(\sigma)} \int_{0}^{s}(s-\tau)^{\sigma-1} \mathcal{F}_{1}(\tau) \psi_{1}\left(\tau, u^{*}(\tau),{ }^{c} D^{\rho}\left(\phi_{p} D^{\beta} v^{*}(\tau)\right)\right) d \tau\right) d s \\
\geq & t^{\beta-1} \max _{t \in J} \int_{0}^{1} \mathcal{G}^{\beta}(1, s) \\
& \times \phi_{q}\left(\frac{1}{\Gamma(\sigma)} \int_{0}^{s}(s-\tau)^{\sigma-1} \mathcal{F}_{1}(\tau) \psi_{1}\left(\tau, u^{*}(\tau),{ }^{c} D^{\rho}\left(\phi_{p} D^{\beta} v^{*}(\tau)\right)\right) d \tau\right) d s
\end{aligned}
$$

implies

$$
u^{*}(t) \geq t^{\beta-1}\left\|u^{*}\right\| \geq \frac{\hbar}{2} t^{\beta-1}>0
$$

Similarly, we get

$$
v^{*}(t) \geq t^{\beta-1}\left\|v^{*}\right\| \geq \frac{\hbar}{2} t^{\beta-1}>0 .
$$

With the help of Lemma 3.2 and $\left(\mathcal{P}_{3}\right)$, the solution $\left(u^{*}, v^{*}\right)$ is nondecreasing for $t \in \mathrm{J}$.

Theorem 3.5 Let hypotheses $\left(\mathbf{H}_{1}\right)$ to $\left(\mathbf{H}_{3}\right)$ be true with $\Delta=\max \left\{\Delta_{1}, \Delta_{2}\right\}<1$, where

$$
\begin{aligned}
& \Delta_{1}=\frac{(q-1) \varrho^{q-1}(2 \beta-\delta) \mathcal{M}_{\psi_{1}}\left\|\mathcal{F}_{1}\right\|}{(\beta-\delta) \Gamma(\beta+1)}\left[\frac{1}{\Gamma(\sigma+1)}+\frac{\mathcal{M}_{\psi_{2}}\left\|\mathcal{F}_{2}\right\|}{\Gamma(\rho+1)}\right] \\
& \Delta_{2}=\frac{(q-1) \varrho^{q-1}(2 \beta-\delta) \mathcal{M}_{\psi_{2}}^{\prime}\left\|\mathcal{F}_{2}\right\|}{(\beta-\delta) \Gamma(\beta+1)}\left[\frac{\left\|\mathcal{F}_{1}\right\| \mathcal{M}_{\psi_{1}}^{\prime}}{\Gamma(\sigma+1)}+\frac{1}{\Gamma(\rho+1)}\right] .
\end{aligned}
$$

Then (1.1) has a unique solution.

Proof Define operator $\Phi=\left(\Phi_{1}, \Phi_{2}\right): \overline{\Omega(r)} / \Omega(r) \rightarrow \mathbf{B}$ by

$$
\Phi(u, v)(t)=\left(\Phi_{1}(u, v), \Phi_{2}(u, v)\right)(t), \quad t \in \mathrm{J},
$$


where

$$
\begin{aligned}
& \Phi_{1}(u, v)(t) \\
& \quad=\int_{0}^{1} \mathcal{G}^{\beta}(t, s) \phi_{q}\left(\frac{1}{\Gamma(\sigma)} \int_{0}^{s}(s-\tau)^{\sigma-1} \mathcal{F}_{1}(\tau) \psi_{1}\left(\tau, u(\tau),{ }^{c} D^{\rho}\left(\phi_{p} D^{\beta} \nu(\tau)\right)\right) d \tau\right) d s
\end{aligned}
$$

and

$$
\begin{aligned}
& \Phi_{2}(u, v)(t) \\
& \quad=\int_{0}^{1} \mathcal{G}^{\beta}(t, s) \phi_{q}\left(\frac{1}{\Gamma(\sigma)} \int_{0}^{s}(s-\tau)^{\sigma-1} \mathcal{F}_{1}(\tau) \psi_{1}\left(\tau, u(\tau),{ }^{c} D^{\rho}\left(\phi_{p} D^{\beta} v(\tau)\right)\right) d \tau\right) d s .
\end{aligned}
$$

Now, for any $(u, v),(\bar{u}, \bar{v}) \in \overline{\Omega(r)} / \Omega(r)$, we have

$$
\begin{aligned}
&\|\Phi(u, v)-\Phi(\bar{u}, \bar{v})\| \\
& \leq \sup _{t \in J} \int_{0}^{1}\left|\mathcal{G}^{\beta}(t, s)\right| \\
& \quad \times\left\{\mid \phi_{q}\left(\frac{1}{\Gamma(\sigma)} \int_{0}^{s}(s-\tau)^{\sigma-1} \mathcal{F}_{1}(\tau) \psi_{1}\left(\tau, u(\tau),{ }^{c} D^{\rho}\left(\phi_{p} D^{\beta} v(\tau)\right)\right) d \tau\right) d s\right. \\
&\left.\quad-\phi_{q}\left(\frac{1}{\Gamma(\sigma)} \int_{0}^{s}(s-\tau)^{\sigma-1} \mathcal{F}_{1}(\tau) \psi_{1}\left(\tau, \bar{u}(\tau),{ }^{c} D^{\rho}\left(\phi_{p} D^{\beta} \bar{v}(\tau)\right)\right) d \tau\right) d s \mid\right\} \\
&+\sup _{t \in J} \int_{0}^{1}\left|\mathcal{G}^{\beta}(t, s)\right| \\
& \quad \times\left\{\mid \phi_{q}\left(\frac{1}{\Gamma(\rho)} \int_{0}^{s}(s-\tau)^{\rho-1} \mathcal{F}_{2}(\tau) \psi_{2}\left(\tau,{ }^{c} D^{\sigma}\left(\phi_{p} D^{\beta} u(\tau)\right), v(\tau)\right) d \tau\right) d s\right. \\
&\left.\quad-\phi_{q}\left(\frac{1}{\Gamma(\rho)} \int_{0}^{s}(s-\tau)^{\rho-1} \mathcal{F}_{2}(\tau) \psi_{2}\left(\tau,{ }^{c} D^{\sigma}\left(\phi_{p} D^{\beta} \bar{u}(\tau)\right), \bar{v}(\tau)\right) d \tau\right) d s \mid\right\} \\
& \leq \frac{(q-1) \varrho^{q-1}(2 \beta-\delta)}{(\beta-\delta) \Gamma(\beta+1)}\left[\frac{\mathcal{M}_{\psi_{1}}\left\|\mathcal{F}_{1}\right\|\|u-\bar{u}\|+\mathcal{M}_{\psi_{1}}^{\prime} \mathcal{M}_{\psi_{2}}^{\prime}\left\|\mathcal{F}_{1}\right\|\left\|\mathcal{F}_{2}\right\|\|v-\bar{v}\|}{\Gamma(\sigma+1)}\right. \\
&\left.+\frac{\mathcal{M}_{\psi_{1}} \mathcal{M}_{\psi_{2}}\left\|\mathcal{F}_{1}\right\| \mathcal{F}_{2}\|\| u-\bar{u}\left\|+\mathcal{M}_{\psi_{2}}^{\prime}\right\| \mathcal{F}_{2}\|\| v-\bar{v} \|}{\Gamma(\rho+1)}\right] \\
& \leq \frac{(q-1) \varrho^{q-1}(2 \beta-\delta) \mathcal{M}_{\psi_{1}}\left\|\mathcal{F}_{1}\right\|}{(\beta-\delta) \Gamma(\beta+1)}\left[\frac{1}{\Gamma(\sigma+1)}+\frac{\mathcal{M}_{\psi_{2}}\|\| \mathcal{F}_{2} \|}{\Gamma(\rho+1)}\right]\|u-\bar{u}\| \\
&+ \Delta_{1}\|u-\bar{u}\|+\Delta_{2}\|v-\bar{v}\| \leq \Delta\|(u, \bar{u})-(v, \bar{v})\| . \\
& \frac{(q-1) \varrho^{q-1}(2 \beta-\delta) \mathcal{M}^{\prime} \psi_{2}\left\|\mathcal{F}_{2}\right\|}{(\beta-\delta) \Gamma(\beta+1)}\left[\frac{\left\|\mathcal{F}_{1}\right\| \mathcal{M}^{\prime}{ }_{\psi_{1}}}{\Gamma(\sigma+1)} \frac{1}{\Gamma(\rho+1)}\right]\|v-\bar{v}\|
\end{aligned}
$$

Thus

$$
\|\Phi(u, v)-\Phi(\bar{u}, \bar{v})\| \leq \Delta\|(u, \bar{u})-(v, \bar{v})\| .
$$

Hence the assumption $\Delta<1$ implies that the operator $\Phi$ is a contraction. Therefore, by Theorem 2.9, (1.1) has a unique fixed point. 


\section{Stability analysis}

In this section, we analyze Hyers-Ulam stability for the proposed problem.

Theorem 4.1 Let assumptions $\left(\mathbf{H}_{1}\right)-\left(\mathbf{H}_{3}\right)$ with $\Delta<1$ hold, along with the condition that the spectral radius of $\mathcal{Q}$ is less than one. Then the solution of (1.1) is Hyers-Ulam stable.

Proof Let $(u, v)$ be the exact and $(\bar{u}, \bar{v})$ be an approximate solution of the considered problem (1.1), then in view of Theorem 3.5 we have

$$
\begin{aligned}
& \|\Phi(u, v)(t)-\Phi(\bar{u}, \bar{v})(t)\| \\
& =\left\|\left(\Phi_{1}(u, v)(t), \Phi_{2}(u, v)(t)\right)-\left(\Phi_{1}(\bar{u}, \bar{v})(t), \Phi_{2}(\bar{u}, \bar{v})(t)\right)\right\| \\
& \leq\left\|\Phi_{1}(u, v)(t)-\Phi_{1}(\bar{u}, \bar{v})\right\|+\left\|\Phi_{2}(u, v)(t)-\Phi_{2}(\bar{u}, \bar{v})\right\| \\
& =\sup _{t \in \mathrm{I}}\left|\Phi_{1}(u, v)(t)-\Phi_{1}(\bar{u}, \bar{v})(t)\right|+\sup _{t \in \mathrm{I}}\left|\Phi_{2}(u, v)(t)-\Phi_{2}(\bar{u}, \bar{v})(t)\right| \\
& =\sup _{t \in \mathrm{J}} \mid \int_{0}^{1} \mathcal{G}^{\beta}(t, s) \phi_{q}\left(\frac{1}{\Gamma(\sigma)} \int_{0}^{s}(s-\tau)^{\sigma-1} \mathcal{F}_{1}(\tau) \psi_{1}\left(\tau, u(\tau),{ }^{c} D^{\rho}\left(\phi_{p} D^{\beta} \nu(\tau)\right)\right) d \tau\right) d s \\
& -\int_{0}^{1} \mathcal{G}^{\beta}(t, s) \phi_{q}\left(\frac{1}{\Gamma(\sigma)} \int_{0}^{s}(s-\tau)^{\sigma-1} \mathcal{F}_{1}(\tau) \psi_{1}\left(\tau, \bar{u}(\tau),{ }^{c} D^{\rho}\left(\phi_{p} D^{\beta} \bar{v}(\tau)\right)\right) d \tau\right) d s \mid \\
& +\sup _{t \in \mathrm{J}} \mid \int_{0}^{1} \mathcal{G}^{\beta}(t, s) \\
& \times \phi_{q}\left(\frac{1}{\Gamma(\rho)} \int_{0}^{s}(s-\tau)^{\rho-1} \mathcal{F}_{2}(\tau) \psi_{2}\left(\tau,{ }^{c} D^{\sigma}\left(\phi_{p} D^{\beta} u(\tau)\right), v(\tau)\right) d \tau\right) d s \\
& -\int_{0}^{1} \mathcal{G}^{\beta}(t, s) \phi_{q}\left(\frac{1}{\Gamma(\rho)} \int_{0}^{s}(s-\tau)^{\rho-1} \mathcal{F}_{2}(\tau) \psi_{2}\left(\tau,{ }^{c} D^{\sigma}\left(\phi_{p} D^{\beta} \bar{u}(\tau)\right), \bar{v}(\tau)\right) d \tau\right) d s \\
& \leq(q-1) \varrho^{q-2} \int_{0}^{1}\left|\mathcal{G}^{\beta}(t, s)\right|\left\{\left(\frac{1}{\Gamma(\sigma)} \int_{0}^{s}(s-\tau)^{\sigma-1}\left\|\mathcal{F}_{1}\right\| \| \psi_{1}\left(\tau, u(\tau),{ }^{c} D^{\rho}\left(\phi_{p} D^{\beta} \nu(\tau)\right)\right)\right.\right. \\
& \left.-\psi_{1}\left(\tau, \bar{u}(\tau),{ }^{c} D^{\rho}\left(\phi_{p} D^{\beta} \bar{v}(\tau)\right)\right) \| d \tau\right) d s \\
& +\frac{1}{\Gamma(\rho)} \int_{0}^{s}(s-\tau)^{\rho-1}\left\|\mathcal{F}_{2}\right\| \| \psi_{2}\left(\tau,{ }^{c} D^{\sigma}\left(\phi_{p} D^{\beta} u(\tau), v(\tau)\right)\right) \\
& \left.-\psi_{2}\left(\tau,{ }^{c} D^{\sigma}\left(\phi_{p} D^{\beta} \bar{u}(\tau), \bar{v}(\tau)\right)\right) \| d \tau d s\right\} \\
& \leq \frac{(q-1) \varrho^{q-1}(2 \beta-\delta)}{(\beta-\delta) \Gamma(\beta+1)}\left[\frac{\mathcal{M}_{\psi_{1}}\left\|\mathcal{F}_{1}\right\|\|u-\bar{u}\|+\mathcal{M}_{\psi_{1}}^{\prime} \mathcal{M}_{\psi_{2}}^{\prime}\left\|\mathcal{F}_{1}\right\|\left\|\mathcal{F}_{2}\right\|\|v-\bar{v}\|}{\Gamma(\sigma+1)}\right. \\
& \left.+\frac{\mathcal{M}_{\psi_{1}} \mathcal{M}_{\psi_{2}}\left\|\mathcal{F}_{1}\right\| \mathcal{F}_{2}\|\| u-\bar{u}\left\|+\mathcal{M}_{\psi_{2}}^{\prime}\right\| \mathcal{F}_{2}\|\| v-\bar{v} \|}{\Gamma(\rho+1)}\right] \\
& \leq \frac{(q-1) \varrho^{q-1}(2 \beta-\delta) \mathcal{M}_{\psi_{1}}\left\|\mathcal{F}_{1}\right\|}{(\beta-\delta) \Gamma(\beta+1) \Gamma(\sigma+1)}\|u-\bar{u}\| \\
& +\frac{(q-1) \varrho^{q-1}(2 \beta-\delta) \mathcal{M}_{\psi_{1}}^{\prime} \mathcal{M}_{\psi_{2}}^{\prime}\left\|\mathcal{F}_{1}\right\|\left\|\mathcal{F}_{2}\right\|}{(\beta-\delta) \Gamma(\beta+1) \Gamma(\sigma+1)}\|v-\bar{v}\| \\
& +\frac{(q-1) \varrho^{q-1}(2 \beta-\delta) \mathcal{M}_{\psi_{1}} \mathcal{M}_{\psi_{2}}\left\|\mathcal{F}_{1}\right\|\left\|\mathcal{F}_{2}\right\|}{(\beta-\delta) \Gamma(\beta+1) \Gamma(\sigma+1)}\|u-\bar{u}\|
\end{aligned}
$$




$$
\begin{aligned}
& +\frac{(q-1) \varrho^{q-1}(2 \beta-\delta) \mathcal{M}_{\psi_{1}}^{\prime}\left\|\mathcal{F}_{1}\right\|}{(\beta-\delta) \Gamma(\beta+1) \Gamma(\sigma+1)}\|v-\bar{v}\| \\
\leq & \|(u, v)-(\bar{u}, \bar{v})\| \mathcal{Q},
\end{aligned}
$$

where $\mathcal{Q}=\left(\begin{array}{ll}\mathcal{C}_{1} & \mathcal{C}_{2} \\ \mathcal{C}_{3} & \mathcal{C}_{4}\end{array}\right)$. Since the spectral radius of $\mathcal{Q}$ is less than one, thus the solution of the considered system (1.1) is Hyers-Ulam stable. Here

$$
\begin{aligned}
& \mathcal{C}_{1}=\frac{(q-1) \varrho^{q-1}(2 \beta-\delta) \mathcal{M}_{\psi_{1}}\left\|\mathcal{F}_{1}\right\|}{(\beta-\delta) \Gamma(\beta+1) \Gamma(\sigma+1)} \\
& \mathcal{C}_{2}=\frac{(q-1) \varrho^{q-1}(2 \beta-\delta) \mathcal{M}_{\psi_{1}}^{\prime} \mathcal{M}_{\psi_{2}}^{\prime}\left\|\mathcal{F}_{1}\right\|\left\|\mathcal{F}_{2}\right\|}{(\beta-\delta) \Gamma(\beta+1) \Gamma(\sigma+1)}, \\
& \mathcal{C}_{3}=\frac{(q-1) \varrho^{q-1}(2 \beta-\delta) \mathcal{M}_{\psi_{1}} \mathcal{M}_{\psi_{2}}\left\|\mathcal{F}_{1}\right\|\left\|\mathcal{F}_{2}\right\|}{(\beta-\delta) \Gamma(\beta+1) \Gamma(\sigma+1)} \\
& \mathcal{C}_{4}=\frac{(q-1) \varrho^{q-1}(2 \beta-\delta) \mathcal{M}_{\psi_{1}}^{\prime}\left\|\mathcal{F}_{1}\right\|}{(\beta-\delta) \Gamma(\beta+1) \Gamma(\sigma+1)}
\end{aligned}
$$

The same approach can be followed to obtain results regarding the generalized HyersUlam, Hyers-Ulam-Rassias, and generalized Hyers-Ulam-Rassias stability.

\section{An illustrative example}

For the support of our theoretical results, an example is presented here.

Example 5.1 Corresponding to (1.1), we consider the system of fractional order differential equations involving $p$-Laplacian operator $\phi_{p}$ as follows:

$$
\left\{\begin{array}{l}
{ }^{c} D^{\sigma}\left(\phi_{p} D^{\beta} u(t)\right)+\frac{t}{\sqrt{1-t}} \frac{u^{2}(t)+1+{ }^{c} D^{\sigma}\left(\phi_{p} D^{\beta} v(t)\right)}{10 e^{t^{2}+1}}=0, \quad t \in[0,1)=\mathrm{J}, \\
{ }^{c} D^{\sigma}\left(\phi_{p} D^{\beta} v(t)\right)+\frac{1}{\sqrt{4-4 t^{2}}} \frac{v(t)+2+{ }^{c} D^{\sigma}\left(\phi_{p} D^{\beta} u(t)\right)}{20+t^{3}}=0, \\
\left(\left[\phi_{p} D^{\beta} u(0)\right]\right)^{(j)}=0, \quad j=0,1,2,3, \ldots, m-1, \\
\left(\left[\phi_{p} D^{\beta} v(0)\right]\right)^{(j)}=0, \\
I^{k-\beta}(u(0))=I^{k-\beta}(v(0))=0, \quad k=2,3, \ldots, m, \\
D^{\delta}(u(1))=D^{\delta}(v(1))=0 .
\end{array}\right.
$$

Set

$$
\psi_{1}\left(t, u(t),{ }^{c} D^{\sigma}\left(\phi_{p} D^{\beta} v(t)\right)\right)=\frac{u^{2}(t)+1+{ }^{c} D^{\sigma}\left(\phi_{p} D^{\beta} u(t)\right)}{10 e^{t^{2}}+1}
$$

and

$$
\psi_{2}\left(t,{ }^{c} D^{\sigma}\left(\phi_{p} D^{\beta} u(t)\right), v(t)\right)=\frac{v(t)+2+{ }^{c} D^{\sigma}\left(\phi_{p} D^{\beta} u(t)\right)}{20+t^{3}} .
$$

Now, for any $u, v, \bar{u}, \bar{v} \in \mathcal{X}$, we have

$$
\left|\psi_{1}(t, u(t), v(t))-\psi_{1}(t, \bar{u}(t), \bar{v}(t))\right| \leq \frac{1}{10 e^{2}}\|u-\bar{u}\|+\frac{1}{10 e^{2}}\|v-\bar{v}\|
$$


and

$$
\left|\psi_{2}(t, u(t), v(t))-\psi_{2}(t, \bar{u}(t), \bar{v}(t))\right| \leq \frac{1}{20}\|u-\bar{u}\|+\frac{1}{20}\|v-\bar{v}\| .
$$

Here, $\mathcal{M}_{\psi_{1}}=\mathcal{M}_{\psi_{1}}^{\prime}=\frac{1}{10 e^{2}}, \mathcal{M}_{\psi_{2}}=\mathcal{M}_{\psi_{2}}^{\prime}=\frac{1}{20}$. Take $q=\frac{5}{2}, \beta=3, \rho=\sigma=\frac{7}{2}, \delta=\frac{3}{2}, \varrho=1$, then $p=\frac{5}{3}$, and upon calculations we have $\Delta=0.000192<1$, so system (5.1) has a unique solution. Further,

$$
\mathcal{H}^{*}=\left(\begin{array}{ll}
0.00002 & 0.00001 \\
0.00001 & 0.00002
\end{array}\right)
$$

and if $\omega_{1}$ and $\omega_{2}$ are the eigenvalues, then $\omega_{1}=0.00001$ and $\omega_{2}=0.00003$. Since the spectral radius of $\mathcal{H}^{*}$ is less than one, thus system (5.1) is Hyers-Ulam stable.

\section{Conclusion}

In this paper, we have utilized the Arzelä-Ascoli theorem, Banach's contraction principle, and Schauder's fixed point theorem to establish existence and uniqueness criteria for the solution of the nonlinear coupled implicit switched singular fractional differential system given in (1.1). Furthermore, under some particular assumptions and conditions, we have proved stability results in the sense of Ulam for the solutions of the said problem. We claim that the approach used to prove the main results is powerful, effectual, and suitable for investigating different qualitative properties of the solutions of nonlinear fractional differential equations.

Funding

The third author would like to thank Prince Sultan University for funding this work through research group Nonlinear Analysis Methods in Applied Mathematics (NAMAM), group number RG-DES-2017-01-17.

Availability of data and materials

Not applicable.

Competing interests

The authors declare that they have no competing interests.

Authors' contributions

All authors contributed equally and significantly to this paper. All authors have read and approved the final version of the manuscript.

\section{Author details}

${ }^{1}$ Department of Mathematics, University of Peshawar, Peshawar, Pakistan. ${ }^{2}$ Department of Mathematics and General Sciences, Prince Sultan University, Riyadh, Saudi Arabia.

\section{Publisher's Note}

Springer Nature remains neutral with regard to jurisdictional claims in published maps and institutional affiliations.

Received: 20 June 2019 Accepted: 4 October 2019 Published online: 16 October 2019

\section{References}

1. Abdeljawad, T., Alzabut, J.: On Riemann-Liouville fractional q-difference equations and their application to retarded logistic type model. Math. Methods Appl. Sci. 41, 8953-8962 (2018)

2. Agarwal, R.P., Baleanu, D., Hedayati, V., Rezapour, S.: Two fractional derivative inclusion problems via integral boundary condition. Appl. Math. Comput. 257, 205-212 (2015)

3. Agarwal, R.P., Zhou, Y., He, Y.: Existence of fractional neutral functional differential equations. Comput. Math. Appl. 59(3), 1095-1100 (2010)

4. Ahmad, N., Ali, Z., Shah, K., Zada, A., Rahman, G.: Analysis of implicit type nonlinear dynamical problem of impulsive fractional differential equations. Complexity 2018, Article ID 6423974, 01-15 (2018) 
5. Ahmad, N., Rafiq, M., Baleanu, D., Rehman, M.A.: Spatio-temporal numerical modeling of auto-catalytic Brusselator model. Rom. J. Phys. 64, 1-14 (2019)

6. Ali, M., Alquran, M., Jaradat, I., Baleanu, D.: Stationary wave solutions for new developed two-waves fifth-order Korteweg-de Vries equation. Adv. Differ. Equ. 2019, 263 (2019)

7. Ali, Z., Zada, A., Shah, K.: On Ulam's stability for a coupled systems of nonlinear implicit fractional differential equations. Bull. Malays. Math. Sci. Soc. 42(5), 2681-2699 (2018)

8. Alkhazzan, A., Jiang, P., Baleanu, D., Khan, H., Khan, A.: Stability and existence results for a class of nonlinear fractional differential equations with singularity. Math. Methods Appl. Sci. 41(18), 9321-9334 (2018)

9. Alzabut, J., Abdeljawad, T.: Nonlinear delay fractional difference equations with applications on discrete fractional Lotka-Volterra competition model. J. Comput. Anal. Appl. 25(5), 889-989 (2018)

10. Aydogan, M.S., Baleanu, D., Mousalou, A., Rezapour, S.: On approximate solutions for two higher-order Caputo-Fabrizio fractional integro-differential equations. Adv. Differ. Equ. 2017, 221 (2017)

11. Aydogan, M.S., Baleanu, D., Mousalou, A., Rezapour, S.: On high order fractional integro-differential equations including the Caputo-Fabrizio derivative. Bound. Value Probl. 2018, 90 (2018)

12. Baghani, H.: Existence and uniqueness of solutions to fractional Langevin equations involving two fractional orders. J. Fixed Point Theory Appl. 20, 63 (2018)

13. Baleanu, D., Ghafarnezhad, K., Rezapour, S.: On a three step crisis integro-differential equation. Adv. Differ. Equ. 2019 $153(2019)$

14. Baleanu, D., Mousalou, A., Rezapour, S.: On the existence of solutions for some infinite coefficient-symmetric Caputo-Fabrizio fractional integro-differential equations. Adv. Differ. Equ. 2017, 145 (2017)

15. Baleanu, D., Mousalou, A., Rezapour, S.: A new method for investigating approximate solutions of some fractional integro-differential equations involving the Caputo-Fabrizio derivative. Adv. Differ. Equ. 2017, 51 (2017)

16. Baleanu, D., Mousalou, A., Rezapour, S.: The extended fractional Caputo-Fabrizio derivative of order $0<\sigma \leq 1$ on $C_{R}[0,1]$ and the existence of solutions for two higher-order series-type differential equations. Adv. Differ. Equ. 2018 255 (2018)

17. Baleanu, D., Rezapour, S., Mohammadi, H.: Some existence results on nonlinear fractional differential equations. Philos. Trans. R. Soc. Lond. A 371, 1-7 (2013)

18. Burton, T., Furumochi, T.: Krasnoselskii's fixed point theorem and stability. Nonlinear Anal. 49(4), 445-454 (2002)

19. Hyers, H.D.: On the stability of the linear functional equation. Proc. Natl. Acad. Sci. 27, 222-224 (1941)

20. Khan, A., Syam, M.I., Zada, A.: Stability analysis of nonlinear fractional differential equations with Caputo and Riemann-Liouville derivatives. Eur. Phys. J. Plus 133, 264 (2018)

21. Khan, R.A., Shah, K.: Existence and uniqueness of solutions to fractional order multi-point boundary value problems. Commun. Appl. Anal. 19, 515-526 (2015)

22. Kilbas, A.A., Srivastava, H.M., Trujillo, J.J.: Theory and Applications of Fractional Differential Equations, North-Holland Mathematics Studies, vol. 204, p. 540. Elsevier, Amsterdam (2006)

23. Kojabad, E.A., Rezapour, S.: Approximate solutions of a sum-type fractional integro-differential equation by using Chebyshev and Legendre polynomials. Adv. Differ. Equ. 2017, 351 (2017)

24. Lakshmikantham, V., Leela, S., Vasundhara, J.D.: Theory of Fractional Dynamic Systems. Cambridge Academic Publishers, Cambridge (2009)

25. Li, Y.: Existence of positive solutions for fractional differential equation involving integral boundary conditions with p-Laplacian operator. Adv. Differ. Equ. 2017, 135 (2017)

26. Liu, Y., Chen, S., Ou, L.: Solvability of Sturm-Liouville boundary value problems for multiple order fractional differential equation. Arab J. Math. Sci. 22, 207-231 (2016)

27. Liu, Z., Lu, L., Szántó, I.: Existence of solutions for fractional impulsive differential equations with $p$-Laplacian operator. Acta Math. Hung. 141(3), 203-219 (2013)

28. Magin, R.L.: Fractional calculus in bioengineering. Crit. Rev. Biomed. Eng. 32(1), 1-104 (2004)

29. Obloza, M.: Hyers stability of the linear differential equation. Rocznik Nauk.-Dydakt. Prace Mat. 13, 259-270 (1993)

30. Oldham, K.B.: Fractional differential equations in electrochemistry. Adv. Eng. Softw. 41(1), 9-12 (2010)

31. Palais, R.S.: A simple proof of Banach contraction principle. J. Fixed Point Theory Appl. 2, 221-223 (2007)

32. Podlubny, I.: Fractional Differential Equations. Academic Press, San Diego (1999)

33. Rassias, T.M.: On the stability of the linear mapping in Banach spaces. Proc. Am. Math. Soc. 72, 297-300 (1978)

34. Rizwan, R., Zada, A.: Nonlinear impulsive Langevin equation with mixed derivatives. Math. Methods Appl. Sci. (2019). https://doi.org/10.1002/mma.5902

35. Rizwan, R., Zada, A., Wang, X.: Stability analysis of non linear implicit fractional Langevin equation with non-instantaneous impulses. Adv. Differ. Equ. 2019, 85 (2019)

36. Royden, H.I.: Real Analysis. Macmillan, New York (1983)

37. Sabatier, J., Agrawal, O.P., Machado, J.A.T.: Advances in Fractional Calculus, Theoretical Developments and Applications in Physics and Engineering. Springer, Dordrecht (2007)

38. Shah, S.O., Zada, A., Hamza, A.E.: Stability analysis of the first order non-linear impulsive time varying delay dynamic system on time scales. Qual. Theory Dyn. Syst. (2019). https://doi.org/10.1007/s12346-019-00315-x

39. Smart, D.R.: Fixed Point Theorems. Cambridge University Press, Cambridge (1980)

40. Urs, C.: Coupled fixed point theorem and application to periodic boundary value problem. Miskolc Math. Notes 14 323-333 (2013)

41. Veeresha, P., Prakasha, D.G., Baleanu, D.: An efficient numerical technique for the nonlinear fractional Kolmogorov-Petrovskii-Piskunov equation. Mathematics 7(3), 1-17 (2019)

42. Wang, J., Lv, L., Zhou, Y.: Ulam stability and data dependence for fractional differential equations with Caputo derivative. Electron. J. Qual. Theory Differ. Equ. 63, 1 (2011)

43. Wang, J., Shah, K., Ali, A.: Existence and Hyers-Ulam stability of fractional nonlinear impulsive switched coupled evolution equations. Math. Methods Appl. Sci. 41,1-11 (2018)

44. Wang, J., Zada, A., Ali, W.: Ulam's-type stability of first-order impulsive differential equations with variable delay in quasi-Banach spaces. Int. J. Nonlinear Sci. Numer. Simul. 19(5), 553-560 (2018)

45. Wang, J., Zada, A., Waheed, H.: Stability analysis of a coupled system of nonlinear implicit fractional anti-periodic boundary value problem. Math. Methods Appl. Sci. (2019). https://doi.org/10.1002/mma.5773 
46. Wu, G.C., Abdeljawad, T., Liu, J., Baleanu, D., Wu, K.T.: Mittag-Leffler stability analysis of fractional discrete-time neural networks via fixed point technique. Nonlinear Anal., Model. Control 24 (2019)

47. Zada, A., Ali, S.: Stability analysis of multi-point boundary value problem for sequential fractional differential equations with non-instantaneous impulses. Int. J. Nonlinear Sci. Numer. Simul. 19(7), 763-774 (2018)

48. Zada, A., Ali, W., Park, C.: Ulam's type stability of higher order nonlinear delay differential equations via integral inequality of Grönwall-Bellman-Bihari's type. Appl. Math. Comput. 350, 60-65 (2019)

49. Zada, A., Mashal, A.: Stability analysis of nth order nonlinear impulsive differential equations in quasi-Banach space. Numer. Funct. Anal. Optim. (2019). https://doi.org/10.1080/01630563.2019.1628049

50. Zada, A., Shaleena, S., Li, T.: Stability analysis of higher order nonlinear differential equations in $\beta$-normed spaces. Math. Methods Appl. Sci. 42(4), 1151-1166 (2019)

Submit your manuscript to a SpringerOpen ${ }^{0}$ journal and benefit from:

- Convenient online submission

- Rigorous peer review

Open access: articles freely available online

- High visibility within the field

- Retaining the copyright to your article

Submit your next manuscript at $\boldsymbol{\nabla}$ springeropen.com 